\title{
REGULARITY PROPERTIES OF CERTAIN FAMILIES OF CHANCE VARIABLES*
}

\author{
BY
}

J. L. DOOB

Let $t$ vary in any simply ordered set, and let $\left\{x_{t}\right\}$ be a family of chance variables. We shall say that the chance variables have the property $\varepsilon$ if whenever $t_{1}<\cdots<t_{n+1}$,

$$
E\left[x_{t_{1}}, \cdots, x_{t_{n}} ; x_{t_{n+1}}\right]=x_{t_{n}}, \dagger
$$

with probability 1 . The subscript $n$ here is any positive integer. It will always be supposed that the given chance variables have expectations, so that the conditional expectations involved in the definition of the property $\varepsilon$ will always exist.

We shall first make a few introductory remarks on the general family of chance variables with the property $\varepsilon$. Then in $\S 1$ it will be supposed that $t$ takes on integral values, and the convergence properties of such sequences of chance variables will be discussed in detail. In $\$ 3$ it will be supposed that $t$ runs through the real numbers. Before discussing this case, it is necessary to investigate the justification for the use of such descriptive terms as continuity, boundedness, and so on, as applied to the random function $x_{t}$. This investigation is made in $\S 2$, in the general case, without the hypothesis of the property $\varepsilon$, and it is found that the above terms can always be made meaningful, and given their usual meanings, at the cost, however, in some cases, of introducing infinite-valued functions.

In the following, we shall always suppose that the $x_{t}$ are measurable functions defined on a space $\Omega$, on certain sets of which a measure function is defined. That this can always be done, and how this is to be done, was shown by Kolmogoroff [5, pp. 27-30]. The space $\Omega$, following Kolmogoroff, will be taken to be the space of real-valued functions of $t$. Integration in terms of probability measure will be denoted by $\int \cdots d P$, and integration will be over all space, unless the domain of integration is otherwise specified. The quali-

\footnotetext{
* Presented to the Society, December 28, 1939, under the title On a certain type of family of chance variables; received by the editors December 11, 1939.

$\dagger$ We shall use the notation $E[y]$ for the expectation of the chance variable $y$, and $E\left[y_{1}, \cdots, y_{n} ; y\right]$ for the conditional expectation of $y$ for given $y_{1}, \cdots, y_{n}$, a function of $y_{1}, \cdots, y_{n}$. If the $y_{j}$ are not finite in number, the notation will be modified accordingly. We shall assume the definitions of Kolmogoroff [5, pp. 41-44] for these conditional expectations. (References in brackets are to the bibliography at the end of the paper.)
} 
fication "with probability 1 " will be used interchangeably with "almost everywhere on $\Omega$." Let $I$ be any set of $t$-values. A $P$-measurable set $\Lambda$ (that is, an $\Omega$-set whose probability is defined) will be said to be an $x_{t}$-set with $t \varepsilon I$, or a set depending only on $t$ for $t$ in $I$, if it is in the Borel field of $\Omega$-sets determined by the sets of the form

$$
\left\{x_{t_{j}}<k_{j}, j=1, \cdots, n\right\}
$$

( $n$ any integer, $k_{1}, \cdots, k_{n}$ any $n$ numbers, $t_{1}, \cdots, t_{n}$ any $n$ distinct $t$-values) or if it differs from an $\Omega$-set in this field by an $\Omega$-set of $P$-measure 0 . If $I$ includes all $t$-values, the $\Omega$-sets in question are all the $P$-measurable sets.

Theorem 0.1. Let $\left\{x_{t}\right\}$ be a family of chance variables with the property $\mathcal{E}$. If $I$ is any $t$-set, and if $t \leqq s_{1}, s_{2}$ for all $t$ in $I$, then

$$
E\left[x_{t}, t \varepsilon I ; x_{s_{1}}\right]=E\left[x_{t}, t \varepsilon I ; x_{s_{2}}\right] \text {, }
$$

with probability 1. If I has a last element $s$, and if $\sigma>s$,

$$
E\left[x_{t}, t \varepsilon I ; x_{\sigma}\right]=x_{s},
$$

with probability 1.

This theorem extends the defining property $\mathcal{E}$, which states the conclusion of Theorem 0.1 for $I$ a finite set. By definition of conditional expectations, $E\left[x_{t}, t \varepsilon I ; x_{s_{j}}\right], j=1,2$, is a function measurable with respect to the $P$-measurable $\Omega$-sets depending only on $x_{t}$ for $t$ in $I$, and such that, if $\Lambda$ is an $\Omega$-set of this type,

$$
\int_{\Lambda} E\left[x_{t}, t \varepsilon I ; x_{s_{j}}\right] d P=\int_{\Lambda} x_{s_{j}} d P, \quad j=1,2 .
$$

Suppose that the $P$-measurable set $\mathrm{M}$ depends only on $t$ for $t=t_{1}, \cdots, i_{n}$, with $t_{j} \varepsilon I, j \leqq n, t_{1}<\cdots<t_{n}$. Then, using the fact that the chance variables $x_{i_{1}}, \cdots, x_{t_{n}}, x_{s_{j}}$ have the property $\varepsilon$,

$$
\int_{\mathbf{M}} x_{s_{j}} d P=\int_{M} E\left[x_{t_{1}}, \cdots, x_{t_{n}} ; x_{s_{j}}\right] d P=\int_{\mathbf{M}} x_{t_{n}} d P, \quad j=1,2 .
$$

Thus

$$
\int_{\mathbf{M}} x_{s_{1}} d P=\int_{\mathbf{M}} x_{s_{2}} d P
$$

and therefore, using (0.1.3),

$$
\int_{M} E\left[x_{t}, t \varepsilon I ; x_{s_{1}}\right] d P=\int_{M} E\left[x_{t}, t \varepsilon I ; x_{s_{2}}\right] d P .
$$


Since any $P$-measurable set which depends only on $t$ for $t \varepsilon I$ can be approximated arbitrarily closely by a set $M,(0.1 .6)$ must hold with the general $\Lambda$ in place of $M$. Since the integrands are measurable with respect to the field of sets $\Lambda$, the integrands are necessarily equal, with probability 1 , as was to be proved. If $I$ has a last element $s, E\left[x_{t}, t \varepsilon I ; x_{s}\right]=x_{s}$, with probability 1 , from the definition of conditional expectations, so in that case both terms of (0.1.1) are equal to $E\left[x_{t}, t \varepsilon I ; x_{s}\right]=x_{s}$, with probability 1 . The last statement of the theorem is merely a rewording of this fact.

THEOREM 0.2. Let $x, y$ be chance variables whose expectations exist, and suppose that $E[x ; y]=x$, with probability 1 . Then $E[x]=E[y]$, and $E|x| \leqq E|y|$.

If $E[x ; y]=x$, with probability 1 , the expectations of the two chance variables $E[x ; y], x$ are equal: $E[y]=E[x]$. Moreover $|x| \leqq E[x ;|y|]$ with probability 1 , and, taking expectations of the two sides of this inequality, we obtain $E|x| \leqq E|y|$.

COROLlaRy. If $\left\{x_{t}\right\}$ is a family of chance variables with the property $\varepsilon$, $E x_{t}$ is independent of $t$, and $E\left|x_{t}\right|$ is a non-decreasing function of $t$.

THEOREM 0.3. Let $\left\{x_{t}\right\}$ be a family of chance variables with the property $\mathcal{E}$. Then for each $t_{0}$, the $x_{t}$ with $t \leqq t_{0}$ are uniformly integrable.*

We show first that

$$
\lim _{k \rightarrow \infty} P\left\{\left|x_{t}\right| \geqq k\right\}=0,
$$

uniformly for $t \leqq t_{0}$. This fact is implied by the following inequality (in which $\left.t \leqq t_{0}\right)$ :

$$
\begin{aligned}
k P\left\{\left|x_{t}\right| \geqq k\right\} & \leqq \int_{\left\{x_{t} \geqq k\right\}} x_{t} d P-\int_{\left\{x_{t} \leqq-k\right\}} x_{t} d P \\
& =\int_{\left\{x_{t} \geqq k\right\}} x_{t_{0}} d P-\int_{\left\{x_{t} \leqq-k\right\}} x_{t_{0}} d P \leqq \int\left|x_{t_{0}}\right| d P .
\end{aligned}
$$

We are using here the fact that if $t \leqq t_{0}, E\left[x_{\ell} ; x_{t_{0}}\right]=x_{t}$ with probability 1 . Now if $t \leqq t_{0}$,

$$
\begin{aligned}
& \int_{\left\{\left|x_{t}\right| \geqq k\right\}}\left|x_{t}\right| d P=\int_{\left\{x_{t} \geqq k\right\}} x_{t} d P-\int_{\left\{x_{t} \leqq-k\right\}} x_{t} d P \\
& =\int_{\left\{x_{t} \geqq k\right\}} x_{t_{0}} d P-\int_{\left\{x_{t} \leqq-k\right\}} x_{t_{0}} d P \leqq \int_{\left\{\left|x_{t}\right| \geqq k\right\}}\left|x_{t_{0}}\right| d P,
\end{aligned}
$$

\footnotetext{
* This means that $\lim _{k \rightarrow \infty} \int\left\{\left|x_{t}\right| \geqq k\right\}\left|x_{t}\right| d P=0$ uniformly in $t$ for $t \leqq t_{0}$.
} 
and, as $k \rightarrow \infty$, the right side goes to 0 uniformly in $t$ (from (0.3.1)), so the left side goes to 0 uniformly in $t$, as was to be proved.

1. Sequences of chance variables with the property $\varepsilon$. We first prove the following theorem.

THEOREM 1.1. Let $\cdots, x_{-1}, x_{0}, \cdots, x$ be chance variables with the property $\varepsilon$. Let $^{*}, n$ be integers with $m \leqq n$, and define the sets $\Lambda, \mathrm{M}$ by

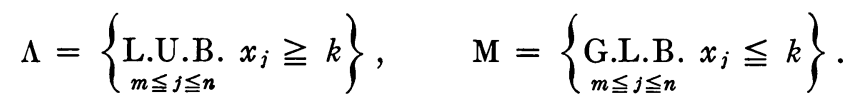

Then if $\mathrm{N}$ is any $P$-measurable $\left(\cdots, x_{m-1}, x_{m}\right)$-set,

$$
\int_{\mathbf{\Lambda} \cdot \mathbf{N}} x d P \geqq k P\{\Lambda \cdot \mathbf{N}\}, \quad \int_{\mathbf{M} \cdot \mathbf{N}} x d P \leqq k P\{\mathrm{M} \cdot \mathrm{N}\} \cdot \dagger
$$

To prove (1.1.1), define the sets $\Lambda_{m}, \cdots$ as follows:

$$
\begin{aligned}
\Lambda_{m} & =\left\{x_{m} \geqq k\right\}, \\
\Lambda_{m+1} & =\left\{x_{m}<k, x_{m+1} \geqq k\right\}, \cdots, \\
\Lambda_{n} & =\left\{x_{m}<k, x_{m+1}<k, \cdots, x_{n-1}<k, x_{n} \geqq k\right\} .
\end{aligned}
$$

Then the $\Lambda_{j}$ are disjunct with sum $\Lambda$, and $\Lambda_{j}$ depends only on $x_{m}, \cdots, x_{j}$. Since $E\left[\cdots, x_{j} ; x\right]=x_{j}$, with probability 1 (Theorem 0.1 ),

$$
\begin{aligned}
\int_{\Lambda \cdot \mathrm{N}} x d P & =\sum_{m}^{n} \int_{\Lambda_{j} \cdot \mathrm{N}} x d P=\sum_{m}^{n} \int_{\Lambda_{j} \cdot \mathrm{N}} x_{j} d P \\
& \geqq k \sum_{m}^{n} P\left(\Lambda_{j} \cdot \mathrm{N}\right)=k P(\Lambda \cdot \mathrm{N}),
\end{aligned}
$$

giving the first inequality of (1.1.1), and the second can be proved in the same way, or by changing the $x_{j}, x$ to their negatives, and applying the result already obtained.

THEOREM 1.2. Let $\cdots, x_{-1}, x_{0}$ be a sequence of chance variables with the property $\mathcal{E}$. Then $\lim _{n \rightarrow-\infty} x_{n}=x$ exists with probability 1 , and the chance variables $x, \cdots, x_{-1}, x_{0}$ have the property $\mathcal{E}$. The chance variables $\left\{x_{j}\right\}$ are uniformly integrable, and $E\left|x_{0}\right| \geqq E\left|x_{-1}\right| \geqq \cdots \geqq E|x| ; E\left|x_{n}\right| \rightarrow E|x|$.

Unless lim $\inf _{n \rightarrow-\infty} x_{n}=\lim \sup _{n \rightarrow-\infty} x_{n}$ with probability 1 , there are numbers $k_{1}, k_{2}$ with $k_{2}>k_{1}$, such that

* The $x_{j}$ may be finite or infinite in number, and if infinite in number may be finite in one direction. The essential fact is that there is a last one $x$.

$\dagger$ The inequalities (1.1.1) are implicit in the work of Ville [9, pp. 100-101] who discussed sequences of non-negative chance variables with the property $\mathcal{E}$. The method of proof we use was used by Lévy $[8$, p. 129], in a related discussion. 


$$
\lim _{n \rightarrow-\infty} \inf x_{n}<k_{1}<k_{2}<\limsup _{n \rightarrow-\infty} x_{n}
$$

with positive probability $\eta$. Let the $\left(\cdots, x_{0}\right)$-set on which $(1.2 .1)$ is true

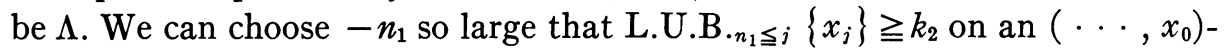
set $\Lambda_{1}$ with $P\left(\Lambda \cdot \Lambda_{1}\right)>\eta\left(1-2^{-1}\right)$. We can choose $n_{2}<n_{1}$ such that

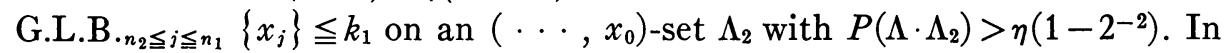
general, if $n_{1}, \cdots, n_{r-1}$ have already been chosen, and if $r$ is odd, we can choose $n_{r}<n_{r-1}$ such that L.U.B. $n_{r} \leqq j \leqq n_{r-1}\left\{x_{j}\right\} \geqq k_{2}$ on an $\left(\cdots, x_{0}\right)$-set $\Lambda_{r}$ with $P\left(\Lambda \cdot \Lambda_{r}\right)>\eta\left(1-2^{-r}\right)$; or if $r$ is even, we can choose $n_{r}<n_{r-1}$ such

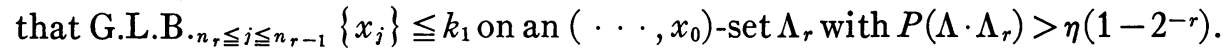
Then if $\Lambda_{n}^{\prime}=\prod_{n}^{\infty} \Lambda_{j}$

$$
P\left(\Lambda_{n}^{\prime}\right)>\eta-\eta 2^{-n}-\eta 2^{-n-1}-\cdots=\eta\left(1-2^{-n+1}\right) .
$$

According to Theorem 1.1, if $r$ is odd,

$$
\int_{\Lambda_{r}^{\prime}} x_{0} d P \geqq k_{2} P\left(\Lambda_{r}^{\prime}\right)
$$

and if $r$ is even

$$
\int_{\Lambda_{r}^{\prime}} x_{0} d P \leqq k_{1} P\left(\Lambda_{r}^{\prime}\right) .
$$

As $r \rightarrow \infty, \Lambda_{r}^{\prime}$ increases to a set $\Lambda^{\prime}$ of measure not less than $\eta$; (1.2.3) and (1.2.4) become

$$
k_{2} P\left(\Lambda^{\prime}\right) \leqq \int_{\Lambda^{\prime}} x_{0} d P \leqq k_{1} P\left(\Lambda^{\prime}\right),
$$

which is impossible, because $k_{1}<k_{2}$. We have thus proved that $\lim _{n \rightarrow-\infty} x_{n}=x$ exists, with probability 1 . According to Theorem 0.3 , the $x_{j}$ are uniformly integrable. Then their limit $x$ is integrable, and term by term integration of $x_{j}$ or $\left|x_{j}\right|$ is legitimate. This means that $E\left|x_{n}\right| \rightarrow E|x|$. The fact that $E\left|x_{0}\right| \geqq E\left|x_{-1}\right| \geqq \cdots$ follows from the corollary to Theorem 0.2 . To show that the chance variables $x, \cdots, x_{-1}, x_{0}$ have the property $\varepsilon$, it is only necessary to show that if $m \leqq n \leqq r \leqq 0, E\left[x, x_{m}, \cdots, x_{n} ; x_{r}\right]=x_{n}$, with probability 1 , and that $E\left[x ; x_{r}\right]=x$, with probability 1 . To prove the first, we must prove that if $\Lambda$ is a set depending only on $x, x_{m}, \cdots, x_{n}$, then

$$
\int_{\Lambda} x_{n} d P=\int_{\Lambda} x_{r} d P .
$$

Now $\Lambda$ evidently depends only on $\cdots, x_{n-1}, x_{n}$; so (1.2.6) follows from the fact that $E\left[\cdots, x_{n} ; x_{r}\right]=x_{n}$, with probability 1 (Theorem 0.1 ). If $\Lambda$ is a set 
depending only on $x,(1.2 .6)$ is true for all $n \leqq r$, and becomes, if $n \rightarrow-\infty$,

$$
\int_{\Delta} x d P=\int_{\Delta} x_{r} d P
$$

that is, $E\left[x ; x_{r}\right]=x$, with probability 1 .

THEOREM 1.3. Let $x_{1}, x_{2}, \cdots$ be a sequence of chance variables with the property $\mathcal{E}$. Then $E\left|x_{1}\right| \leqq E\left|x_{2}\right| \leqq \cdots$. If $\lim _{n \rightarrow \infty} E\left|x_{n}\right|=l<\infty$, then $\lim _{n \rightarrow \infty} x_{n}=x$ exists, with probability 1 , and $E|x| \leqq l$. If the $x_{j}$ are uniformly integrable, $\lim _{n \rightarrow \infty} x_{n}=x$ exists, with probability 1 , and the chance variables $x_{1}, x_{2}, \cdots, x$ have the property $\varepsilon$.

Ville has studied sequences of non-negative chance variables with the property $\mathcal{E}$. Since, by the corollary to Theorem 0.2 , Ville's hypotheses imply that

$$
E x_{1}=E x_{2}=\cdots=E\left|x_{1}\right|=E\left|x_{2}\right|=\cdots,
$$

the hypotheses of the first part of Theorem 1.3 are satisfied, in Ville's case. Ville proved* that in his case L.U.B. ${ }_{j \geqq 1}\left|x_{j}\right|<\infty$, with probability 1 (implied by our conclusion that $\lim _{n \rightarrow \infty} x_{n}$ exists with probability 1 , and that the limit is integrable), and applied this fact to the study of certain games of chance.

Proof of Theorem 1.3. According to the corollary to Theorem 0.2, $E\left|x_{n}\right|$ is monotone non-decreasing. Suppose that $\lim _{n \rightarrow \infty} E\left|x_{n}\right|=l<\infty$. Unless $\lim _{\inf _{n \rightarrow \infty}} x_{n}=\lim \sup _{n \rightarrow \infty} x_{n}$, with probability 1 , there are numbers $k_{1}, k_{2}$, with $k_{1}<k_{2}$, such that

$$
\liminf _{n \rightarrow \infty} x_{n}<k_{1}<k_{2}<\limsup _{n \rightarrow \infty} x_{n}
$$

with positive probability $\eta$. Let the $\left(x_{1}, x_{2}, \cdots\right)$-set on which this is true be $\Lambda$. We can choose $n_{1}$ so large that L.U.B. ${ }_{j \leqq n_{1}}\left\{x_{j}\right\} \geqq k_{2}$ on an $\left(x_{1}, x_{2}, \cdots\right)$-set $\Lambda_{1}$ with $P\left(\Lambda \cdot \Lambda_{1}\right)>\eta\left(1-3^{-1}\right)$. We can then choose $n_{2}>n_{1}$ so large that G.L.B. $n_{1} \leqq j \leqq n_{2}\left\{x_{j}\right\} \leqq k_{1}$ on an $\left(x_{1}, x_{2}, \cdots\right)$-set $\Lambda_{2}$ with $P\left(\Lambda \cdot \Lambda_{2}\right)>\eta\left(1-3^{-2}\right)$. In general if $n_{1}, \cdots, n_{r-1}$ have already been chosen, and if $r$ is odd, we can find

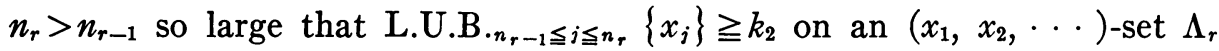
with $P\left(\Lambda \cdot \Lambda_{r}\right)>\eta\left(1-3^{-r}\right)$; or if $r$ is even, we can find $n_{r}>n_{r-1}$ so large that G.L.B. $n_{r-1} \leqq j \leqq n_{r}\left\{x_{j}\right\} \leqq k_{1}$ on an $\left(x_{1}, x_{2}, \cdots\right)$-set $\Lambda_{r}$ with $P\left(\Lambda \cdot \Lambda_{r}\right)>\eta\left(1-3^{-r}\right)$. Then if $\Lambda_{n}{ }^{\prime}=\prod_{1}^{n} \Lambda_{j}$,

$$
P\left(\Lambda_{n}^{\prime}\right)>\eta-\eta / 3-\eta / 3^{2}-\cdots=\eta / 2 .
$$

According to Theorem 1.1, if $m \geqq n_{2 r}$,

\footnotetext{
* $[9$, chaps. $4,5$.
} 


$$
\int_{\Lambda_{2 r}^{\prime}} x_{m} d P \leqq k_{1} P\left(\Lambda_{2 r}^{\prime}\right), \quad \int_{\Lambda_{2 r-1}^{\prime}} x_{m} d P \geqq k_{2} P\left(\Lambda_{2 r-1}^{\prime}\right)
$$

Then if $\mathrm{M}_{r}=\Lambda_{2 r-1}^{\prime}-\Lambda_{2 r}$

$$
\int_{\mathbf{M}_{r}} x_{m} d P \geqq k_{2} P\left(\Lambda_{2 r-1}^{\prime}\right)-k_{1} P\left(\Lambda_{2 r}^{\prime}\right)=\left(k_{2}-k_{1}\right) P\left(\Lambda_{2 r-1}^{\prime}\right)+k_{1} P\left(\mathrm{M}_{r}\right) .
$$

Now the $\mathbf{M}_{r}$ are disjunct, so if $m$ is sufficiently large (depending on $q$ ),

$$
\begin{aligned}
\sum_{r=1}^{q} \int_{\mathrm{M}_{r}} x_{m} & \geqq\left(k_{2}-k_{1}\right) \sum_{r=1}^{q} P\left(\Lambda_{2 r-1}^{\prime}\right)+k_{1} \sum_{r=1}^{q} P\left(\mathrm{M}_{r}\right) \\
& \geqq \frac{q\left(k_{2}-k_{1}\right)}{2} \eta-\left|k_{1}\right| .
\end{aligned}
$$

But this is impossible, since then

$$
l \geqq \int\left|x_{m}\right| d P \geqq \sum_{r=1}^{q} \int_{\mathbf{M}_{r}} x_{m} d P \geqq \frac{q \eta}{2}\left(k_{2}-k_{1}\right)-\left|k_{1}\right|
$$

that is,

$$
l \geqq(1 / 2) q \eta\left(k_{2}-k_{1}\right)-\left|k_{1}\right|,
$$

for all $q$. We have thus proved that $\lim _{n \rightarrow \infty} x_{n}=x$ exists with probability 1 . By Fatou's lemma, $E|x| \leqq l$, and the fact that $E|x|<\infty$ implies that $x$ is finite, with probability 1 . If the $x_{j}$ are supposed uniformly integrable, it follows that the sequence $\left\{E\left|x_{j}\right|\right\}$ is uniformly bounded. Then $\lim _{n \rightarrow \infty} x_{n}=x$ exists with probability 1 . Because of the uniform integrability, term by term integration of the sequence $\left\{x_{n}\right\}$ is allowed on any $P$-measurable set, and we can readily verify that the sequence of chance variables $x_{1}, x_{2}, \cdots, x$ has the property $\varepsilon$. In fact, all that need be proved is that $E\left[x_{\alpha_{1}}, \cdots, x_{a_{n}} ; x\right]=x_{\alpha_{n}}$ with probability 1 , if $\alpha_{1}<\cdots<\alpha_{n}$. This will follow if it can be shown that whenever $\Lambda$ is an $\left(x_{1}, x_{2}, \cdots\right)$-set depending only on $x_{\alpha_{1}}, \cdots, x_{\alpha_{n}}$, then

$$
\int_{\Lambda} x d P=\int_{\Lambda} x_{\alpha_{n}} d P
$$

Since the chance variables $x_{1}, x_{2}, \cdots$ have the property $\varepsilon,(1.3 .8)$ is true if $x$ is replaced by $x_{m}$ with $m>\alpha_{n}$. Then if $m \rightarrow \infty$, we obtain (1.3.8).

COROLLARY. Let $y_{1}, y_{2}, \cdots$ be a sequence of chance variables whose expectations exist, and suppose that $E\left[y_{1}, \cdots, y_{n} ; y_{n+1}\right]=0, n=1,2, \cdots$, with probability 1. Then $E\left|\sum_{1}^{n} y_{j}\right|$ is monotone non-decreasing with $n$, and if $\lim _{n \rightarrow \infty} E\left|\sum_{1}^{n} y_{j}\right|<\infty$, the series $\sum_{1}^{\infty} y_{j}$ is convergent, with probability 1 . 
For if $x_{n}=\sum_{1}^{n} y_{j}, n=1,2, \cdots$, the $x_{n}$ have the property $\varepsilon$, and Theorem 1.3 is applicable, giving the result of the corollary.

Lévy [8, pp. 247-248] has discussed series of chance variables $\sum_{1}^{\infty} y_{j}$, with $E\left[y_{1}, \cdots, y_{n} ; y_{n+1}\right]=0, n=1,2, \cdots$, with probability 1 , relating the convergence of the given series to that of their conditional dispersions.

An important particular case of this corollary is that in which the $y_{j}$ are mutually independent. The hypothesis on the conditional expectations becomes in this case the hypothesis that $E y_{1}=E y_{2}=\cdots=0$, and the corollary has been deduced in this case. ${ }^{*}$ In this special case it has also been shown that the partial sums $\left\{x_{j}\right\}$ are necessarily uniformly bounded by a chance variable whose expectation exists. It follows that in this case the $x_{i}$ are always uniformly integrable.

The following example shows that the hypotheses of the first part of Theorem 1.3 do not imply that the sequence $x_{1}, x_{2}, \cdots, x$ has the property $\varepsilon$. Let $x_{0}=1$ with probability 1 . Let $x_{1}=1 / 2$ with probability $\left(1-4^{-1}\right)$ and $x_{1}=5 / 2$ with probability $1 / 4$. In general if $x_{1}, \cdots, x_{r}$ have already been determined, we determine $x_{r+1}$ as follows. The values of $x_{r+1}$ depend only on those of $x_{r}$, and if $x_{r}=a$, we set $x_{r+1}=2^{-r-1}$ with probability $\left(1-4^{-r-1}\right)$ and $x_{r+1}=\xi$ with probability $4^{-r-1}$, choosing $\xi$ to make the expectation of $x_{r+1}$ (with $x_{r}=a$ ) equal to 0 :

$$
\frac{\xi}{4^{r+1}}+\frac{1}{2^{r+1}}\left(1-\frac{1}{4^{r+1}}\right)=a .
$$

Then it is easily verified that $x_{n}=2^{-n}$ with probability $\left(1-4^{-n}\right)$, if $n \geqq 1$, so that $x_{n} \rightarrow x=0$, with probability 1 . The chance variables are all positive and the hypotheses of the first part of Theorem 1.3 are satisfied. The sequence $x_{1}, x_{2}, \cdots, x$ certainly does not have the property $\varepsilon$.

The following theorem is essentially the converse of the second part of Theorem 1.3.

TheOREM 1.4. Let $x_{1}, x_{2}, \cdots, x^{\prime}$ be chance variables with the property $\mathcal{E}$. Then

(a) $\lim _{n \rightarrow \infty} x_{n}=E\left[x_{1}, x_{2}, \cdots ; x^{\prime}\right]$ with probability 1 ;

(b) the chance variables $x_{1}, x_{2}, \cdots, x, x^{\prime}$ have the property $\varepsilon$;

(c) if $x^{\prime}$ is a function of $x_{1}, x_{2}, \cdots, \lim _{n \rightarrow \infty} x_{n}=x^{\prime}$, with probability 1.

Lévy [8, p. 129] proved part (c) of this theorem, under the hypothesis that $x$ only takes on the values 1 and 0 . His proof can be extended to cover Theorem 1.4. He puts the result in the following form (we are dropping his restriction on the values taken on by $\left.x^{\prime}\right)$; let $y_{1}, y_{2}, \cdots$ be a sequence of

* Doob [2]. 
chance variables and let $x^{\prime}$ be a chance variable dependent upon them. Then if $x_{n}=E\left[y_{1}, \cdots, y_{n} ; x^{\prime}\right]$, he proves that $x_{n} \rightarrow x^{\prime}$ with probability 1 . Since $x_{1}, x_{2}, \cdots, x^{\prime}$ have the property $\varepsilon$, this is exactly our Theorem $1.4(\mathrm{c})$.

Proof of (a), (b). According to Theorem 0.3 , the $x_{j}$ are uniformly integrable. Then Theorem 1.3 states that $\lim _{n \rightarrow \infty} x_{n}=x$ exists with probability 1 , and that the chance variables $x_{1}, \cdots, x$ have the property $\varepsilon$. If we show that the chance variables $x_{1}, \cdots, x, x^{\prime}$ have the property $\varepsilon$, it will follow (because of Theorem 0.1 ) that $x=E\left[x_{1}, \cdots ; x^{\prime}\right]$ with probability 1 . To show that the chance variables $x_{1}, \cdots, x, x^{\prime}$ have the property $\varepsilon$ it is sufficient to show that if $\alpha_{1}<\cdots<\alpha_{n}$, then $E\left[x_{\alpha_{1}} \cdots, x_{\alpha_{n}}, x ; x^{\prime}\right]=x$ with probability 1 , that is, that if $\Lambda$ is any set depending on $x_{\alpha_{1}}, \cdots, x_{\alpha_{n}}, x$,

$$
\int_{\Lambda} x^{\prime} d P=\int_{\Lambda} x d P
$$

Now $\Lambda$ is a set depending on $x_{1}, x_{2}, \cdots$ (because $x_{n} \rightarrow x$ with probability 1 ). Equation (1.4.1) is certainly true for $\Lambda$ depending only on a finite number of the $x_{j}$ (because if $x_{\nu}$ is the last of these $x_{j}$, both integrals are equal to the integral of $x_{\nu}$ over $\Lambda: x_{1}, \cdots, x^{\prime}$ and $x_{1}, \cdots, x$ have the property $\varepsilon$ ). Then (1.4.1) is true in the general case because the general $\Lambda$ can be approximated arbitrarily closely by the special ones.

Proof of (c). If $x^{\prime}$ is a function of $x_{1}, x_{2}, \cdots, E\left[x_{1}, x_{2}, \cdots ; x^{\prime}\right]=x^{\prime}$ with probability 1 , so that $x=x^{\prime}$ with probability 1 .

Theorem 1.5. Let $x_{1}, x_{2}, \cdots, x$ be chance variables with the property $\varepsilon$. Suppose that $E|x \log | x||<\infty$. There is then a number $K$ depending only on $E|x \log | x||$, and such that $E\left[\right.$ L.U.B. $\left.n \geqq 1\left|x_{n}\right|\right]<K$. 1.1 ,

Let $\xi=$ L.U.B. ${ }_{n \geqq 1}\left\{x_{n}\right\}$, and let $\Lambda=\{\xi \geqq k\}, k>0$. According to Theorem

$$
\int_{\Lambda} x d P \geqq k P(\Lambda)
$$

Now

$$
\int_{\Lambda \cdot\{|x|<k / 2\}}|x| d P \leqq \frac{k}{2} P(\Lambda)
$$

so

$$
\int_{\{|x| \geqq k / 2\}}|x| d P \geqq \int_{\Lambda \cdot\{|x| \geqq k / 2\}} x d P \geqq \frac{k}{2} P(\Lambda)=\frac{k}{2} P\{\xi \geqq k\} .
$$

This inequality, together with the fact that $E|x \log | x||<\infty$, has been 
used in an analogous discussion (Wiener [10, pp. 17-18]) to show that $E \xi \leqq K_{1}$, where $K_{1}$ only depends on $E|x \log | x||$. Applying this result to $-x_{1},-x_{2}, \cdots,-x$, completes the proof of the theorem.

We have seen above in the discussion of the corollary to Theorem 1.3 that if the $x_{j}$ are the partial sums of a series of mutually independent chance variables having zero expectations, the hypothesis that $E|x \log | x||<\infty$ is unnecessary in Theorem 1.5.

2. One-parameter families of chance variables. In this section we shall consider a one-parameter family of chance variables $\left\{x_{t}\right\},-\infty<t<\infty$, or $0 \leqq t<\infty$. The probability relations are those derived from certain elementary probabilities: if $t_{1}, \cdots, t_{n}$ are distinct $t$-values, and if $k_{1}, \cdots, k_{n}$ are any real numbers, $P\left\{x_{t_{j}}<k_{j}, j \leqq n\right\}$ is given. The mathematical setup appropriate to the study of the probability relations of the $x_{t}$ is the following. Let $\Omega^{*}$ be the space of all real-valued functions of $t$. A probability measure is determined on $\Omega^{*}$ by the measures of certain elementary sets, which we shall call neighborhoods: a set $\Lambda$ of functions $x(t)$ satisfying

$$
a_{j}<x\left(t_{j}\right)<b_{j}, \quad j=1, \cdots, n,-\infty \leqq a_{j}<b_{j} \leqq+\infty,
$$

will be called a neighborhood, and we define a measure of $\Lambda, P^{*}(\Lambda)$, setting

$$
P^{*}(\Lambda)=P\left\{a_{j}<x_{t_{j}}<b_{j}, j \leqq n\right\} . \dagger
$$

If $x_{s}(\omega)$ is the function of $\omega \varepsilon \Omega^{*}$ which for $\omega=\omega_{0}: x_{0}(t)$ assumes the value $x_{0}(s)$ (so that we can write, somewhat loosely, $x_{s}(\omega)=x(s)$ ), $x_{s}(\omega)$ is a $P^{*}$-measurable function ( $s$ fixed), and the probability relations of the $\left\{x_{t}\right\}$ become measure relations of the corresponding $\left\{x_{t}(\omega)\right\}$. The outer measure $\bar{P}^{*}(\Lambda)$ of an $\Omega^{*}$-set $\Lambda$ is defined as the lower limit of $P^{*}\left(\Lambda^{\prime}\right)$ where $\Lambda^{\prime} \supset \Lambda$ and $\Lambda^{\prime}$ is $P^{*}$-measurable. $\ddagger$ The inner measure of an $\Omega^{*}$-set $\Lambda$ is defined as $1-\bar{P}^{*}\left(\Lambda_{1}\right)$, where $\Lambda_{1}$ is the complement of $\Lambda$. It is usually convenient to use a subspace $\Omega$ of $\Omega^{*}$ (with $\bar{P}^{*}(\Omega)=1$ ) instead of $\Omega^{*}$ itself. The $P^{*}$-measure on $\Omega^{*}$ determines $P$ measure on $\Omega$, and

$$
P^{*}\left\{x\left(t_{j}\right)<k_{j}, j=1, \cdots, n\right\}=P\left\{x\left(t_{j}\right)<k_{j}, j=1, \cdots, n\right\} .
$$

We are using $x\left(t_{j}\right)$ to denote the $P$-measurable function $x_{t_{j}}(\omega)$, which in turn represents the original chance variable $x_{t_{j}}$, and we shall continue to use this notation where there is no danger of confusion. The symbol $x(t)$ thus may represent either a point $\omega$ of $\Omega^{*}$, that is, a function of $t$, or, if we are fixing $t$, a function of $\omega: x_{t}(\omega)$.

In discussions of continuity and related questions, it is desirable to con-

$\dagger$ Cf. Doob [1, pp. 107-110].

$\ddagger$ We can even suppose that $\Lambda^{\prime}$ is restricted to be a denumerable sum of neighborhoods. 
sider L.U.B.t $\varepsilon_{I} x_{t}$, G.L.B.t $\varepsilon_{I} x_{t}$, where $I$ is a $t$-interval. It was pointed out by Kolmogoroff $\left[5\right.$, p. 26] that the probability that L.U.B.t $\varepsilon_{I} x_{t} \leqq k$ or that G.L.B. $\varepsilon_{\varepsilon} x_{t} \leqq k$ cannot be derived from the given probabilities. Since we are giving a complete treatment of the problem here, we shall prove a somewhat stronger result, pointed out to the writer by Dr. P. R. Halmos.

Theorem 2.1. Let $\Omega^{*}$ and $P^{*}$-measure be defined as explained above. Then the $\omega$-set $\left\{\right.$ L.U.B. $\left.{ }_{t_{I}} x(t) \leqq k\right\}$ has inner measure 0 , for any nondenumerable set $I$ and real number $k$.

This theorem shows that the $\omega$-set $\left\{\right.$ L.U.B.t $\left.{ }_{\varepsilon} x(t) \leqq k\right\}$, if it is $P^{*}$-measurable, must have measure 0 , irrespective of the $P^{*}$-measure. If the complement of the $\omega$-set in question is $\Lambda$, it will be sufficient to show that $\bar{P}^{*}(\Lambda)=1$. The $\Omega^{*}$-set $\Lambda$ is the set of all $x(t)$ with $x\left(t_{0}\right)>k$ for some $t_{0}$ in $I$. Let $\Gamma \boldsymbol{\sigma} \Omega^{*}$ be any denumerable sum of neighborhoods, with $\Gamma \supset \Lambda$. It will be sufficient to show that $P^{*}(\Gamma)=1$, and in fact we shall show that $\Gamma=\Omega^{*}$. Let $t_{1}, t_{2}, \cdots$ be the $t$-values used in defining the $\Gamma_{j}$. Let $x_{0}(t)$ be any element of $\Omega^{*}$. There is surely a function $x_{1}(t)$, with $x_{1}\left(t_{j}\right)=x_{0}\left(t_{j}\right)$ for all $j$, and such that for some $s$ in $I$, $x_{1}(s)>k$. Then $x_{1}(t) \varepsilon \Lambda \subset \Gamma$, so $x_{0}(t) \varepsilon \Gamma$ also, because $x_{1}\left(t_{j}\right)=x_{0}\left(t_{j}\right)(j \geqq 1)$. Thus $\Gamma=\Omega^{*}$, as was to be proved.

Two ways have been suggested, to define $P\left\{\right.$ L.U.B. $\left.\varepsilon_{\varepsilon} x_{t} \leqq k\right\}$ and $P\left\{\right.$ G.L.B. $\left.{ }_{t} \varepsilon{ }_{I} x_{t} \leqq k\right\}$. Khintchine [4, pp. 68-69] considers a sequence $\left\{t_{j}\right\}$ of $t$-values in $I$. The probability $P\left\{\right.$ L.U.B. $\left.j_{\geqq 1} x_{t_{j}} \leqq k\right\}$ is already determined, and Khintchine defines $P\left\{\right.$ L.U.B.t $\left.\varepsilon_{I} x_{t} \leqq k\right\}$ as the greatest lower bound of these probability numbers, for all denumerable sets $\left\{t_{j}\right\}$ in $I ; P\left\{\right.$ G.L.B. $\left.{ }_{t} \varepsilon_{I} x_{t} \leqq k\right\}$ can be defined similarly. With this definition, the "L.U.B." in "L.U.B. t $_{I} x_{t}$ " is not taken literally, necessarily not, since $x_{t}$ is not a single function of $t$ and since the problem has not been reduced to one of studying functions of $t$. The second way is to find a subspace $\Omega$ of $\Omega^{*}$, of outer measure 1 , such that there is a denumerable set $\left\{s_{j}\right\}$ for which, if $x(t) \varepsilon \Omega$, and if $I$ is an open interval,

$$
\underset{t \varepsilon I}{\text { L.U.B. }} x(t)=\underset{s_{j} \varepsilon I}{\text { L.U.B. }} x\left(s_{j}\right), \quad \text { G.L.B. } x(t)=\underset{t \varepsilon I}{\text { G.L.B. }} x\left(s_{j}\right) \text {. }
$$

If there is such an $\Omega$, the $x(t)$-set $\left\{\right.$ L.U.B. I $\left._{I} x(t) \leqq k\right\}$ becomes a $P$-measurable set:

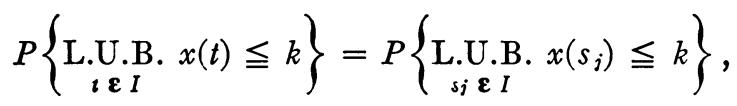

and similarly for the G.L.B. Evidently the two methods of definition give the same numerical values to $P\left\{\right.$ L.U.B. $\left.{ }_{t} \varepsilon_{I} x_{t} \leqq k\right\}$. It must be stressed that in (2.1.2) the set $\left\{\right.$ L.U.B.t $\left.\varepsilon_{I} x(t) \leqq k\right\}$ is a subset of $\Omega$, whose $P$-measure is equal 
to that of the set on the right since the sets are identical. The $P$-measure of the set on the right is equal to the $P^{*}$-measure of the $\Omega^{*}$-set $\left\{\right.$ L.U.B. s $\left._{j} \varepsilon_{I} x\left(s_{j}\right) \leqq k\right\}$, by the definition of $P$-measure, and may be any number between 0 and 1 , depending on $k$ and the particular characteristics of the given $P^{*}$-measure. On the other hand, Theorem 2.1 shows that the $\Omega^{*}$-set $\left\{\right.$ L.U.B.t $\left.\varepsilon_{I} x(t) \leqq k\right\}$ necessarily has inner measure 0 .

A process with a space $\Omega$ as just described is called quasi-separable. Let $I$ be any open $t$-interval (of length $|I|$ ) containing the point $s$. Define $x_{*}(s)$, $x^{*}(s)$ by

$$
x_{*}(s)=\lim _{|I| \rightarrow 0} \underset{s_{j} \varepsilon \boldsymbol{\varepsilon} I}{\text { G.L.B. }} x\left(s_{j}\right), \quad x^{*}(s)=\lim _{|I| \rightarrow 0} \text { L.U.B. } s_{j} \boldsymbol{\varepsilon} I
$$

Then the condition that $\Omega$ be the space of a quasi-separable process is equivalent to the condition that if $x(t) \varepsilon \Omega$, and if $\left\{s_{j}\right\}$ is an everywhere dense denumerable $t$-set,

$$
x_{*}(t) \leqq x(t) \leqq x^{*}(t)
$$

for all $t$. $\uparrow$ It has been shown (Doob [1]) that many given probability measures on $\Omega^{*}$ are such that there is a quasi-separable process. It will be shown below that every probability measure has this property, if infinite-valued functions are allowed. Applications will then be made in $\$ 3$ to $P^{*}$-measures in which the chance variables $\left\{x_{t}\right\}$ have the property $\mathcal{E}$.

In order to treat the problems below it will sometimes be necessary to allow our functions $x(t)$ to take on the values $\pm \infty$. Kolmogoroff's proof [5, pp. 27-30] that the elementary given probabilities determine a probability measure needs no change to cover this case. All distributions will be finite: for each $t,|x(t)|<\infty$ with probability 1 . It is easily shown that the set of everywhere finite-valued functions $x(t)$ has outer measure 1 , and inner measure 0 .

Let $I$ be an open $t$-interval, and let $\left\{t_{j}\right\}$ be a sequence in $I$. Then L.U.B. $j_{\geqq 1} x\left(t_{j}\right)$ is a chance variable, that is, a $P^{*}$-measurable function on $\Omega^{*}$. We shall show below that there is a sequence $\left\{\bar{t}_{j}\right\}$ in $I$ such that if $\left\{t_{j}\right\}$ is any sequence in $I$,

$$
\xi=\underset{j \geqq 1}{\text { L.U.B. }} x\left(\bar{t}_{j}\right) \geqq \underset{j \geqq 1}{\text { L.U.B. }} x\left(t_{j}\right)
$$

with probability 1 . The (possibly infinite-valued) chance variable $\xi$ will be called the generalized upper bound of $x(t)$ in $I$, and will be denoted by $U_{I}[x(t)]$. The generalized lower bound $L_{I}[x(t)]$ is defined in a similar fashion. To demonstrate the existence of the $\left\{\bar{t}_{j}\right\}$, we note that the values of

† Cf. Doob [1, pp. 110-111]. 
$E\left[\operatorname{arc} \tan\right.$ L.U.B. $\left.{ }_{i \geqq 1} x\left(t_{j}\right)\right] \dagger$ for $\left\{t_{j}\right\}$ a sequence in $I$, have a least upper bound M. There are sequences $\left\{t_{j}^{(k)}\right\}, k=1,2, \cdots$, such that

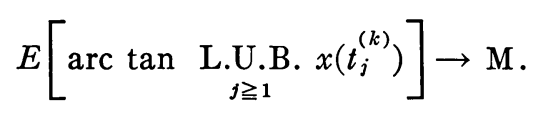

Let $\bar{t}_{1}, \bar{t}_{2}, \cdots$ be the $t_{j}^{(\boldsymbol{k})}, j, k \geqq 1$, arranged in some order. Then evidently

$$
E\left[\arctan \underset{j \geqq 1}{\text { L.U.B. }} x\left(\bar{t}_{j}\right)\right]=\mathrm{M} \text {. }
$$

Now let $\left\{t_{j}\right\}$ be any sequence in $I$. We shall show that it is impossible that L.U.B. ${ }_{j \geqq 1} x\left(t_{j}\right)>$ L.U.B. ${ }_{j \geqq 1} x\left(\bar{t}_{j}\right)$ with positive probability, that is, on an $\Omega^{*}$-set of positive $P^{*}$-measure. If this inequality were possible with positive probability, the inequality would still be true if the sequence $\left\{t_{j}\right\}$ were augmented to include the $\bar{t}_{j}$. Then, supposing this has been done,

$$
\arctan \underset{j \geqq 1}{\text { L.U.B. }} x\left(t_{j}\right) \geqq \arctan \underset{j \geqq 1}{\text { L.U.B. }} x\left(\bar{t}_{j}\right)
$$

for all $\omega$, and there is actual inequality with positive probability. This implies that

$$
E\left[\arctan \underset{j \geqq 1}{\text { L.U.B. }} x\left(t_{j}\right)\right]>E\left[\arctan \underset{j \geqq 1}{\text { L.U.B. }} x\left(\bar{t}_{j}\right)\right]=\mathrm{M},
$$

contradicting the definition of $\mathbf{M}$. Thus the $\bar{t}_{j}$ have the required property. Evidently $U_{I}[x(t)]$ is not a uniquely determined function, but is determined up to an $\Omega^{*}$-set of $P^{*}$-measure 0 , in the sense that two generalized upper bounds are equal almost everywhere on $\Omega^{*}$.

THEовем 2.2. There is a sequence $\left\{s_{j}\right\}$, independent of the open interval $I$, such that, for each $I$,

$$
\underset{s_{j} \varepsilon I}{\text { L.U.B. }} x\left(s_{j}\right)=U_{I}[x(t)], \quad \underset{s_{i} \varepsilon I}{\text { G.L.B. }} x\left(s_{j}\right)=L_{I}[x(t)],
$$

with probability 1.

We have seen that to each $I$ correspond sequences $\left\{t_{j}^{\prime}\right\},\left\{t_{j}^{\prime \prime}\right\}$, such that

$$
\text { L.U.B. } x\left(t_{j}^{\prime}\right)=U_{I}[x(t)], \quad \text { G.L.B. } x\left(t_{j}^{\prime \prime}\right)=L_{I}[x(t)],
$$

with probability 1 . Let $\left\{s_{j}\right\}$ be any sequence including all these $t_{j}^{\prime}, t_{j}^{\prime \prime}$, for every $I$ with rational end points. Then evidently (2.2.1) is true, with probability 1 , if $I$ is such an interval. Let $I$ be any open $t$-interval, and let $I_{1}, I_{2}, \ldots$

$\dagger$ We define $\operatorname{arc} \tan \pm \infty$ as $\pm \pi / 2$. 
be intervals with rational end points, such that $I_{1} \subset I_{2} \subset \cdots$ and that $\sum_{1}^{\infty} I_{j}=I$. Then

$$
\underset{s_{j} \varepsilon I_{n}}{\operatorname{L.U.B.}} x\left(s_{j}\right)=U_{I_{n}}[x(t)],
$$

with probability 1 . Let $\left\{\sigma_{j}\right\}$ be a sequence of $t$-values, including the $s_{j}$. This inclusion implies that

$$
\underset{s_{j} \varepsilon I_{n}}{\text { L.U.B. }} x\left(s_{j}\right)=U_{I_{n}}[x(t)]=\underset{\sigma_{j} \varepsilon I_{n}}{\text { L.U.B. }} x\left(\sigma_{j}\right)
$$

with probability 1 . Let the $\left\{\sigma_{j}\right\}$ also be so inclusive that

$$
U_{I}[x(t)]=\underset{\sigma_{j} \varepsilon I}{\operatorname{L.U} I} \operatorname{x}\left(\sigma_{j}\right)
$$

with probability 1 . Then if $n \rightarrow \infty$ in (2.2.4), we find that

$$
\underset{s_{j} \varepsilon I}{\text { L.U.B. }} x\left(s_{j}\right)=\underset{\sigma_{j} \varepsilon I}{\text { L.U.B. }} x\left(\sigma_{j}\right)
$$

with probability 1 , and (2.2.5), (2.2.6) taken together imply the first part of (2.2.1). The second part is proved in the same way.

Lемма 2.3. If a sequence $\left\{s_{j}\right\}$ is chosen as described in Theorem 2.2, and if $x^{*}(t), x_{*}(t)$ are the upper and lower limiting functions of $x(t)$ on $\left\{s_{j}\right\}$, as defined in (2.1.3), then for each value of $t$,

$$
x_{*}(t) \leqq x(t) \leqq x^{*}(t)
$$

with probability 1 .

For if $x^{*}\left(s_{0}\right)<x\left(s_{0}\right)$ for some $t$-value $s_{0}$, on an $\Omega^{*}$-set of positive measure, and if $I$ is an open interval containing $s_{0}$,

$$
U_{I}[x(t)]=\underset{j \geqq 1}{\text { L.U.B. }} x\left(s_{j}\right)<x\left(s_{0}\right)
$$

on an $\Omega^{*}$-set of positive measure, if $I$ is sufficiently small, contradicting the definition of $U_{I}[x(t)]$. Thus the second half of the inequality is proved, and the first is proved in the same way.

Corollary. For each value of $t, x_{*}(t)<\infty, x^{*}(t)>-\infty$, with probability 1.

It has not been necessary, as yet, to utilize a subspace $\Omega$ of $\Omega^{*}$, rather than $\Omega^{*}$ itself. As Theorem 2.1 shows, such a transition is necessary, in discussing quasi-separable processes.

TheOREM 2.4. Whatever the given $P^{*}$-measure, there is a space $\Omega$ of a quasiseparable process. In some cases it is necessary to allow infinite-valued functions in $\Omega$. 
This theorem states that we can find a space on which the generalized upper and lower bounds become actual upper and lower bounds. It will be utilized to show that a regularity property of $x(t)$ known to be true when $t$ is restricted to denumerable sets implies the same type of regularity for all $t$, for the functions of a suitably chosen space $\Omega$. (Compare Theorems $2.7,2.8$, 2.9.)

Proof of Theorem 2.4. Let $\left\{s_{j}\right\}$ be a sequence as described in Theorem 2.2. Define $x_{*}(t), x^{*}(t)$ as in (2.1.3). Let $\Omega$ be the space of all $x(t)$ with

$$
x_{*}(t) \leqq x(t) \leqq x^{*}(t)
$$

identically in $t$. If $x_{*}(t)=+\infty$, this means that $x(t)=+\infty$, and if $x^{*}(t)=-\infty$, this means that $x(t)=-\infty$. To prove the theorem, we need only show that $\bar{P}^{*}(\Omega)=1$. Let $\Gamma=\sum_{1}^{\infty} \Gamma_{n}$ be any denumerable sum of neighborhoods with $\Gamma \supset \Omega$. It will be sufficient to show that $P^{*}(\Gamma)=1$. Let $t_{1}, t_{2}, \cdots$ be the $t$-values used in defining the $\Gamma_{j}$. Let $\Omega_{1}$ be the space of all $x(t)$ satisfying (2.4.1) for $t=t_{1}, t_{2}, \cdots$. Then by Lemma $2.3, P^{*}\left(\Omega_{1}\right)=1$, so it will be sufficient to prove that $\Gamma \supset \Omega_{1}$. Let $\xi(t)$ be any element in $\Omega_{1}$. We show that $\xi(t) \varepsilon \Gamma$. We have, since $\xi(t) \varepsilon \Omega_{1}$,

$$
\xi_{*}\left(t_{j}\right) \leqq \xi\left(t_{j}\right) \leqq \xi^{*}\left(t_{j}\right), \quad j=1,2, \cdots .
$$

Now there is an $x_{0}(t)$ in $\Omega$ such that $x_{0}\left(t_{j}\right)=\xi\left(t_{j}\right), j \geqq 1$ : we need only define $x_{0}(t)$ so that

$$
\xi_{*}(t) \leqq x_{0}(t) \leqq \xi^{*}(t)
$$

$t \notin\left\{t_{j}\right\}$.

Then $x_{0}(t) \varepsilon \Omega \subset \Gamma$ so $\xi(t)$ is in $\Gamma$ (because $\xi\left(t_{j}\right)=x\left(t_{j}\right), j \geqq 1$ ), as was to be proved. An example will be given below to show that the introduction of infinite-valued functions may be essential in some cases. Evidently it must be known that $x^{*}(t),-x_{*}(t)$ are (simultaneously for all $t$ ) not equal to $+\infty$, with probability 1 , if infinite-valued functions are to be avoided.

We pass to the discussion of measurable processes. A process with space $\Omega$ is said to be measurable if the function $x_{s}(\omega)(=x(s))$ is measurable as a function of the two variables $s, \omega$, with $\omega \varepsilon \Omega$.†

THEOREM 2.5. Let $P^{*}$-measure be such that there is a measurable process. Then there is a space $\Omega$ of a quasi-separable measurable process. In some cases it is necessary to allow infinite-valued functions in $\Omega$.

Define the function $\phi_{n}(t)$ by

$$
\begin{array}{r}
\phi_{n}(t)=k 2^{-n}, \quad \text { if } \quad(k-1) 2^{-n}<t \leqq k 2^{-n}, \quad k=0, \pm 1, \cdots, \\
n=1,2, \cdots
\end{array}
$$

† Cf. Doob [1, p. 113]. 
Then since there is a measurable process with the given $P^{*}$-measure, there is a constant $c$, and a sequence of integers $\left\{a_{n}\right\}$, such that for $t$ fixed, not in some set $e$ of Lebesgue measure 0 ,

$$
\lim _{n \rightarrow \infty} x\left[c+\phi_{a_{n}}(t)\right]=x(c+t),
$$

with probability $1 . \dagger$ Let $\left\{\sigma_{j}\right\}$ be the sequence of all numbers $\left\{c+k 2^{-n}\right\}$, $k=0, \pm 1, \cdots, n=1,2, \cdots$. Define $x^{* *}(t), x_{* *}(t)$ by

$$
x^{* *}(t)=\limsup _{n \rightarrow \infty} x\left[c+\phi_{a_{n}}(t)\right], \quad x_{* *}(t)=\liminf _{n \rightarrow \infty} x\left[c+\phi_{a_{n}}(t)\right] .
$$

Then for each $t$ not in $e, x_{* *}(t)=x(t)=x^{* *}(t)$, with probability 1 . Let $\left\{s_{j}\right\}$ be a sequence of numbers with the properties described in Theorem 2.2. We can suppose that the sequence $\left\{s_{j}\right\}$ includes the $\left\{\sigma_{j}\right\}$, since any sequence including the sequence $\left\{s_{j}\right\}$ also has the properties described in Theorem 2.2. Defining $x_{*}(t), x^{*}(t)$ as in (2.1.3),

$$
x_{*}(t) \leqq x_{* *}(t) \leqq x^{* *}(t) \leqq x^{*}(t),
$$

and for each $t$, we have seen that

$$
x_{*}(t) \leqq x(t) \leqq x^{*}(t),
$$

with probability 1 . Let $\Omega$ be the set of all functions $x(t)$ satisfying

$$
x_{* *}(t) \leqq x(t) \leqq x^{* *}(t)
$$

for $t \notin e$, and (2.5.5) for $t \varepsilon e$. (We are allowing $x(t)$ to take on infinite values.) Then if $x(t) \varepsilon \Omega, x(t)$ satisfies (2.5.5) identically in $t$. This means, according to the definition of quasi-separability, that if $\bar{P}^{*}(\Omega)=1, \Omega$ is the space of a quasi-separable process. To prove the theorem, it will therefore be sufficient to prove that $\bar{P}^{*}(\Omega)=1$ and that the function $x_{8}(\omega)$ is a measurable function of $(s, \omega),(\omega \varepsilon \Omega)$. Let $\Gamma=\sum_{1}^{\infty} \Gamma_{n}$ be any denumerable sum of neighborhoods with $\Gamma \supset \Omega$. To show that $\bar{P}^{*}(\Omega)=1$, we shall show that $P^{*}(\Gamma)=1$. Let $t_{1}, t_{2}, \cdots$ be the $t$-values used in defining the neighborhoods. Let $\Omega$ be the space of all $x(t)$ such that (2.5.6) is true for the $t_{j} \varepsilon e$, and (2.5.5) for the $t_{j} \varepsilon e$. Then $P^{*}\left(\Omega_{1}\right)=1$, so it will be sufficient to show that $\Gamma \supset \Omega_{1}$. Let $\xi(t)$ be any function in $\Omega_{1}$ :

$$
\begin{aligned}
\xi_{* *}\left(t_{j}\right) & \leqq \xi\left(t_{j}\right) \leqq \xi^{* *}\left(t_{j}\right), & & t_{j} \xi e, \\
\xi_{*}\left(t_{j}\right) & \leqq \xi\left(t_{j}\right) \leqq \xi^{*}\left(t_{j}\right), & & t_{j} \varepsilon e .
\end{aligned}
$$

Now there is an $x_{0}(t)$ in $\Omega$ such that $x_{0}\left(t_{j}\right)=\xi\left(t_{j}\right), j \geqq 1$ : we need only have $x_{0}(t)$ satisfy 


$$
\xi_{* *}(t) \leqq x_{0}(t) \leqq \xi^{* *}(t),
$$

$t \notin\left\{t_{j}\right\}$.

Then $x_{0}(t) \varepsilon \Omega \subset \Gamma$. This means that $\xi(t) \varepsilon \Gamma$ (because $x_{0}\left(t_{j}\right)=\xi\left(t_{j}\right), j \geqq 1$ ). Then $\Gamma \supset \Omega_{1}: P^{*}(\Gamma)=1$. To complete the proof we must show that $x_{s}(\omega)$ is $(s, \omega)$ measurable $(\omega \varepsilon \Omega)$. It is readily verified that $x\left[c+\phi_{a_{n}}(s)\right]$, which is $x_{\sigma}(\omega)$ with $\sigma=c+\phi_{a_{n}}(s)$ is $(s, \omega)$-measurable, and it follows that $x_{s}^{* *}(\omega), x_{8 * *}(\omega)$ (defined in terms of $x^{* *}(t), x_{* *}(t)$ as $x_{s}(\omega)$ is in terms of $\left.x(t)\right)$ are $(s, \omega)$-measurable. Then since if $s \notin e$, and if $\omega \varepsilon \Omega$ (rewriting (2.5.6))

$$
x_{s * *}(\omega) \leqq x_{s}(\omega) \leqq x_{s}^{* *}(\omega),
$$

and since the extremes are equal ( $s \notin e$ ) for almost all $\omega \varepsilon \Omega$, the extremes are equal for almost all $(s, \omega)$. Then $x_{s}(\omega)=x_{s}^{* *}(\omega)=x_{s * *}(\omega)$ for almost all $(s, \omega)$ $(\omega \varepsilon \Omega)$, and so $x_{s}(\omega)$ is $(s, \omega)$-measurable $(\omega \varepsilon \Omega)$, as was to be proved.

COROLLARY. The hypotheses of Theorem 2.5 imply the following stronger conclusion. There is a space $\Omega$ of a stochastic process, and a denumerable everywhere dense $t$-set $\left\{s_{j}\right\}$ such that, defining $x_{*}(t), x^{*}(t)$ by (2.1.3), and $y_{*}(t), y^{*}(t) b y$

$$
y_{*}(t)=\liminf _{s_{j} \downarrow t} x\left(s_{j}\right), \quad y^{*}(t)=\limsup _{s_{j} \downarrow t} x\left(s_{j}\right),
$$

if $x(t) \varepsilon \Omega$,

$$
x_{*}(t) \leqq x(t) \leqq x^{*}(t)
$$

for all $t$, and, except for a $t$-set $e_{1}$ (independent of the function $x(t)$ ) of Lebesgue measure 0 ,

$$
y_{*}(t) \leqq x(t) \leqq y^{*}(t) .
$$

The set $e_{1}$ contains no point $t^{\prime}$ with the property that whenever $\left\{t_{n}\right\}$ is a sequence converging to $t^{\prime}$ from above: $t_{n} \downarrow t^{\prime}$, then

$$
\lim _{n \rightarrow \infty} \inf x\left(t_{n}\right) \leqq x\left(t^{\prime}\right) \leqq \lim _{n \rightarrow \infty} \sup x\left(t_{n}\right)
$$

with probability 1.

The proof of Theorem 2.5 actually proves most of the corollary. Using the notation of that proof, we have

$$
y_{*}(t) \leqq x_{* *}(t) \leqq x^{* *}(t) \leqq y^{*}(t) .
$$

This means that, to prove the corollary, we need only modify the definition of $\Omega$, as follows. The space $\Omega$ is now to include all functions $x(t)$ satisfying (2.5.6) for all $t \notin e$, as before, and also for $t=t^{\prime} \varepsilon e$, if $t^{\prime}$ is as described in the statement of the corollary; otherwise $x(t)$ is to satisfy (2.5.5) for $t \varepsilon e$. The proof then goes through essentially as before. 
The following example of a stochastic process illustrates the application of the preceding theorem. We shall restrict $t$ to be non-negative. Define $P^{*}$ measure as follows. Let $x(0)=0$ with probability 1 . The chance variable $x(t+l)-x(t)(l>0)$ has a distribution taking on only integral values, and

$$
P^{*}\{x(t+l)-x(t)=\nu\}=\frac{e^{-l} l^{\nu}}{\nu !}, \quad \nu=0,1,2, \cdots .
$$

If $0 \leqq t_{0}<\cdots<t_{n}$, the chance variables

$$
x\left(t_{1}\right)-x\left(t_{0}\right), \cdots, x\left(t_{n}\right)-x\left(t_{n-1}\right)
$$

are to be mutually independent. According to Theorem 2.4, there is a space $\Omega$ of a quasi-separable process. Let $\left\{s_{j}\right\}$ be the denumerable set involved in the definition of quasi-separability, so that (2.1.4) is true for all $t$, if $x(t) \varepsilon \Omega$. We can assume that $t=0 \varepsilon\left\{s_{j}\right\}$. Evidently $x(t)$, considering $t$ only in $\left\{s_{j}\right\}$ is monotone non-decreasing, starting from 0 , and increasing only by jumps of unit magnitude, with probability 1 . Then except for an $\Omega$-set of $P$-measure 0 , the functions in $\Omega$ are monotone non-decreasing, and continuous except for jumps of magnitude 1 , these jumps being the only points of increase. It is easily verified that $\Omega$ is the space of a measurable process. $\dagger$ According to the corollary to Theorem 2.5 , there is even a space $\Omega_{1}$ of a stochastic process all of whose functions are continuous on the right. In fact the set $e_{1}$ of that corollary is empty, because for any $t, t_{n} \downarrow t$ implies that $x\left(t_{n}\right) \rightarrow x(t)$ with probability 1 . In this case infinite-valued functions need not be introduced, but we shall use this example to derive another, in which the introduction of infinitevalued functions is essential, in discussing quasi-separable processes. For each $x(t) \varepsilon \Omega_{1}$, as defined above, we define a function $\xi(t)$, to be 0 at $t=t_{0}$ if $x(t)$ has a jump there, and otherwise to be $1 /\left|t_{1}-t_{0}\right|$, where $t_{1}$ is the closest point to $t_{0}$ where there is a jump. The probability relations for the $\xi(t)$ are derived from those of the $x(t)$. For each $t, \xi(t)=\xi_{t}(\omega)$ is a $P$-measurable function, and the one-parameter family of chance variables $\left\{\xi_{t}(\omega)\right\}$ determines a new stochastic process. The particular functions $\xi(t)$ we have defined above form a space $\Omega_{\xi}$, which can be shown to be of outer measure 1 in terms of the new probability measure, $\ddagger$ but we shall not need this fact. Evidently if $\xi(t) \varepsilon \Omega_{\xi}, \xi(t)$ is continuous except at an isolated denumerable set. If $\left\{s_{j}\right\}$ is any everywhere dense denumerable set, and if $\xi_{*}(t), \xi^{*}(t)$ are defined as usual, $\xi_{*}(t)=+\infty$ at some point in any given interval of length $l$ with probability $\left(1-e^{-l}\right)$. Thus

$$
P\left\{\max _{0 \leqq t<\infty} \xi_{*}(t)=+\infty\right\}=1
$$

$\dagger$ This fact also follows from Theorem 2.5 (ii) of Doob [1].

$\ddagger$ Ambrose-Doob [3]. 
and the introduction of infinite-valued functions is necessary to obtain a quasi-separable process for the $\xi(t)$. The space $\Omega_{\xi}$ is not that of a quasi-separable process, but it can be shown that if each $\xi(t)$ in $\Omega_{\xi}$ is altered to $+\infty$ where it vanishes, the resulting $\xi(t)$ are the functions of a quasi-separable process.

Theorem 2.6. Suppose that there is a $t$-set $E$ such that whenever $t_{n} \downarrow t \varepsilon E$ $x\left(t_{n}\right)$ approaches a limit in probability. Then there is a denumerable set $D \subset E$ such that if $t \varepsilon E-D, x(t+h) \rightarrow x(t)$ in probability as $h \rightarrow 0$.

Define a metric on the space of $P^{*}$-measurable functions $f(\omega)$, by defining the distance between $f_{1}$ and $f_{2}$ to be

$$
d\left(f_{1}, f_{2}\right)=\int\left|\arctan f_{1}-\arctan f_{2}\right| d P
$$

so that $d\left(f, f_{n}\right) \rightarrow 0$ means $f_{n} \rightarrow f$ in probability, and conversely, if $f$ is finitevalued. Then for each $t$, the $\omega$-function $x_{t}(\omega)$ becomes a point of this space, and as $t$ varies we obtain a function of $t$. This function of $t, \Phi(t)$, has the property that if $t \varepsilon E, \lim _{t_{n} \downarrow t} \Phi\left(t_{n}\right)$ exists. Then if $t \varepsilon E, \lim _{t^{\prime} \downarrow t} \Phi\left(t^{\prime}\right)$ exists. It is well known that a function with this property can have at most a denumerable number of discontinuities on $E$, and this set of discontinuities is the $D$ of the theorem.

Theorem 2.7. Suppose that there is a $t$-set $E$ such that whenever $t_{n} \downarrow t \varepsilon E$ $\lim _{n \rightarrow \infty} x\left(t_{n}\right)$ exists, with probability 1 . Then there is a denumerable set $D^{\prime} \subset E$, such that if $t \varepsilon E-D^{\prime}, t_{n} \rightarrow t$ implies that $x\left(t_{n}\right) \rightarrow x(t)$ with probability 1 . If $\Omega$ is the space of a quasi-separable process, each $x(t)$ in $\Omega$ is continuous almost everywhere on $E$ with probability 1 , and if $t_{0} \varepsilon E-D^{\prime}, x(t)$ is continuous at $t_{0}$, with probability $1 . \dagger$

Since convergence almost everywhere on $\Omega^{*}$ implies convergence in measure (that is, in probability) on $\Omega^{*}$, Theorem 2.6 implies that there is a denumerable $t$-set $D \subset E$, such that $t \varepsilon E-D, t_{n} \downarrow t$ implies that $x\left(t_{n}\right) \rightarrow x(t)$ in probability, and since $\lim _{n \rightarrow \infty} x\left(t_{n}\right)$ exists with probability 1 , this limit must be $x(t)$. Let $S$ be an everywhere dense denumerable $t$-set. The hypothesis of Theorem 2.7 implies that if $t \varepsilon E-D, \lim _{t^{\prime} \downarrow t} x\left(t^{\prime}\right)$ exists with probability 1 , if $t^{\prime}$ is restricted to lie in $S$. $\ddagger$ Let $\nu$ be any positive integer. Then if $t_{0} \varepsilon E-D$, there is an open interval $I\left(t_{0}\right)$ with $t_{0}$ as left-hand end point, such that the oscillation of $x(t)(t \varepsilon S)$ on $I\left(t_{0}\right)$ is not greater than $1 / \nu$, neglecting an $\Omega^{*}$-set of measure not greater than $1 / \nu$. The intervals $I(t)$ cover $E$ except possibly

$\dagger$ Both in this theorem and in the preceding one, convergence to an infinite limit can be allowed. The definition of convergence in probability to an infinite limit is made in an obvious way.

$\ddagger$ Doob [1, p. 111]. 
for an at most denumerable set $D^{(\nu)}$ : for the $I(t)$ with $t \varepsilon E$, and $t$ not covered, are nonoverlapping intervals, and so are at most denumerable. Moreover if $t_{0} \varepsilon E-D^{(\nu)}$, the oscillation of $x(t)(t \varepsilon S)$ at $t_{0}$ is not greater than $1 / \nu$, neglecting a possible $\Omega^{*}$-set (depending on $t_{0}$ ) of measure not greater than $1 / \nu$. Then if $t_{0} \varepsilon E-D_{1}$, where $D_{1}=\sum_{1}^{\infty} D^{(v)}$, the oscillation of $x(t)(t \varepsilon S)$ is 0 , neglecting a possible $\Omega^{*}$-set of measure 0 . This means that if $t_{0} \varepsilon E-D^{\prime}$, where $D^{\prime}=D+D_{1}$, $\lim _{t \rightarrow t_{0}} x(t)=x\left(t_{0}\right)(t \varepsilon S)$, with probability 1 . Now let $\Omega$ be the space of a quasiseparable process (Theorem 2.4 states that there always is such a space), and let $S$ be a set of $t$-values such that (2.1.4) is satisfied for all $t$. It follows that if $t \varepsilon E-D^{\prime}, \dagger x(t)$ (in $\Omega$ ) is continuous at $t$, with probability 1 . This means, in particular, that if $t_{n} \rightarrow t \varepsilon E-D^{\prime}$, then $x\left(t_{n}\right) \rightarrow x(t)$ with probability $1 \mathrm{in} \Omega$, and therefore in $\Omega^{*}$. The theorem will be completely proved when it is shown that $x(t)$ (in $\Omega$ ) is continuous almost everywhere in $E$, with probability 1 . To show this, let $x_{s}^{*}(\omega)\left[x_{s *}(\omega)\right]$ be the function of $\omega: x(t)$ which for $\omega=\omega_{0}: x_{0}(t)$ takes on the value $x_{0}^{*}(s)\left[x_{0 *}(s)\right]$. We can then write $x_{s}^{*}(\omega)=x^{*}(s), x_{s *}(\omega)=x_{*}(s)$. The functions $x_{s}^{*}(\omega), x_{s *}(\omega)$ are easily seen to be measurable functions of $(s, \omega)$ for $\omega \varepsilon \Omega$, and for $s \varepsilon E-D^{\prime}$ are equal almost everywhere on $\Omega$. Then by Fubini's theorem, they are equal almost everywhere on $E-D^{\prime}$ (that is, almost everywhere on $E$ ) for almost all $\omega: x(t)$. The equality of $x^{*}\left(t_{0}\right), x_{*}\left(t_{0}\right)$ means the continuity of $x(t)$ at $t_{0}$; so the theorem is now completely proved.

Theorem 2.8. Suppose that $P^{*}$-measure has the property that, whatever the denumerable set $S$, almost every $x(t)$ in $\Omega^{*}$ has the property that $\lim _{t \cdot t_{0}} x(t)(t \varepsilon S)$ exists for all $t_{0}$. Let $D_{1}\left(D_{2}\right)$ be the $t$-set on which it is not true that $t_{n} \downarrow t\left(t_{n} \uparrow t\right)$ implies that $x\left(t_{n}\right) \rightarrow x(t)$ with probability 1 . Then $D_{1}, D_{2}$ are denumerable. Let $\Omega$ be the space of a quasi-separable process. Then for almost all $x(t)$ in $\Omega$, $\lim _{h \downarrow 0} x(t+h)$ exists, for all $t$, and $x(t)$ is continuous except perhaps for a denumerable number of discontinuities (varying with the function $x(t)$ in $\Omega$ ). There is a quasi-separable process with space $\Omega_{1}$, for which, in addition to the above, $x(t)$ in $\Omega_{1}$ is continuous on the right: $x(t+0)=x(t)$ for all $t \notin D_{1}$.

The hypotheses imply that if $t_{n} \downarrow t, \lim _{n \rightarrow \infty} x\left(t_{n}\right)$ exists with probability 1 . Then according to Theorem 2.7, $D_{1}$ and $D_{2}$ are denumerable. Let $\Omega$ be the space of a quasi-separable process, and let $S$ be a sequence of $t$-values such that (2.1.4) is satisfied for all $t$. Then since, according to the hypotheses, $\lim _{h \downarrow 0} x^{*}(t+h), \lim _{h \downarrow 0} x_{*}(t+h)$ exist and are equal for all $t$, with probability 1 , it follows that if $x(t) \varepsilon \Omega$ and is not in an exceptional $\omega$-set of probability 0 , $\lim _{h \downarrow 0} x(t+h)$ exists, for all $t$. A function with this property has at most a denumerable number of discontinuities. According to the corollary to Theorem 2.5 there must be a space $\Omega_{1}$, of a stochastic process, that is, with

$\dagger$ The set $D^{\prime}$ depends on $S$. 
$\bar{P}^{*}\left(\Omega_{1}\right)=1$, all of whose elements are functions continuous on the right if $t \notin D_{1}$.

This theorem, which sounds somewhat clumsy, has been phrased with the applications in mind. It is applicable, for example, to any differential process: one in which if $t_{0}<t_{1}<\cdots<t_{n}$, the chance variables

$$
x_{t_{1}}-x_{t_{0}}, \cdots, x_{t_{n}}-x_{t_{n-1}}
$$

are mutually independent. $†$ The first example given above was one of such a process.

THEOREM 2.9. Suppose that $P^{*}$-measure has the property that, whatever the denumerable $t$-set $S$, almost every $x(t)$ in $\Omega^{*}$ coincides on $S$ with an everywhere continuous function $f_{\omega}(t)$. Then there is a space $\Omega$ of a (quasi-separable) process, whose functions $x(t)$ are everywhere continuous.

According to Theorem 2.4, there is a quasi-separable process. Evidently almost all the functions involved in this process are everywhere continuous, so the continuous functions themselves can be taken as the functions of a process.

In many applications, the following situation arises. There is given a $P^{*}$-measure, and a one-parameter family of $P^{*}$-measurable functions $\left\{f_{t}(\omega)\right\}$, $-\infty<t<+\infty$. Let $\Omega_{y}^{*}$ be the space of all functions $y(t)$. We define a $P^{*}$ measure on $\Omega_{y}^{*}$ by setting

$$
P_{y}^{*}\left\{y\left(t_{j}\right)<k_{j}, j=1, \cdots, n\right\}=P^{*}\left\{f_{t_{j}}(\omega)<k_{j}, j=1, \cdots, n\right\} .
$$

In other words, we use the family of chance variables $\left\{f_{t}(\omega)\right\}$ to define a new stochastic process. The $y(t)$ of $\Omega_{y}^{*}$ are the elements of a stochastic process whose measure relations are those given by the $x(t)$. It is frequently desirable to restrict the $x(t)$ to some space $\Omega$ (to obtain a quasi-separable or measurable process) and to consider only the $y(t)$ given by

$$
y(t)=f_{t}(\omega), \quad \omega \varepsilon \Omega,-\infty<t<\infty .
$$

It is known that the space $\Omega_{y}$ of these $y(t)$ has outer $P_{y}^{*}$-measure 1 , and so is the space of a stochastic process, $\ddagger$ but it is also desirable to know that this process has further properties, such as quasi-separability or measurability. In the application to be made in the next section, $f_{t}(\omega)$ is only determined, for each $t$, up to an $\omega$-set of measure 0 , and this is the usual case. We can then vary $\Omega_{y}$ by changing $f_{t}(\omega)$ for each $t$ on an $\omega$-set of measure 0 . We shall show that by doing this we can (i) make $\Omega_{y}$ the space of a quasi-separable process, or

† Cf. Doob [1, pp. 134-137]; the theorems of the present paper make possible considerable simplification of this paper.

$\ddagger$ Ambrose-Doob [3]. 
(ii) (if the $P_{y}^{*}$-measure is such that there is a measurable process) we can make $\Omega_{y}$ the space of a quasi-separable measurable process.

Proof of (i). Let $\left\{s_{n}\right\}$ be a $t$-set with the properties described in Theorem 2.2, using $P_{y}^{*}$-measure. Define $f_{t *}(\omega), f_{t}^{*}(\omega)$ by

$$
f_{t *}(\omega)=\lim _{|I| \rightarrow 0} \text { G.L.B. } f_{s_{j}} f_{s_{j}}(\omega), \quad f_{t}^{*}(\omega)=\lim _{|I| \rightarrow 0} \underset{s_{j} \varepsilon I}{\text { L.U.B. }} f_{s_{j}}(\omega),
$$

where $I$ is an open interval, containing $t$, of length $|I|$. Then according to Lemma 2.3, for each $t$,

$$
f_{t *}(\omega) \leqq f_{t}(\omega) \leqq f_{t}^{*}(\omega)
$$

almost everywhere on $\Omega$. There is a subspace $\Omega_{0} \subset \Omega$ of $P$-measure 1 , such that these inequalities are satisfied for all $\omega \varepsilon \Omega_{0}$ if $t \varepsilon\left\{s_{j}\right\}$. If $f_{t_{0}}\left(\omega_{0}\right)$ does not satisfy this inequality for $\omega_{0} \varepsilon \Omega_{0}$, redefine $f_{t_{0}}\left(\omega_{0}\right)$ as any number between $f_{t_{0} *}\left(\omega_{0}\right)$ and $f_{t_{0}}^{*}\left(\omega_{0}\right)$. We thus change $f_{t_{0}}(\omega)$ on at most an $\omega$-set of measure 0 , and incidentally may introduce some infinite values. The new function $g_{t}(\omega)$ has the properties

$$
\begin{gathered}
g_{s_{j}}(\omega)=f_{s_{j}}(\omega), \\
f_{t *}(\omega)=g_{t *}(\omega) \leqq g_{t}(\omega) \leqq g_{t}^{*}(\omega)=f_{t}^{*}(\omega) .
\end{gathered}
$$

Let $g_{t}(\omega)=0$ if $\omega \varepsilon \Omega-\Omega_{0}$. Then the functions $y(t): y(t)=g_{t}(\omega), \omega \varepsilon \Omega$, determine a space $\Omega_{y}$ of the desired quasi-separable process.

The proof of (ii) is along the same lines, and will be omitted. The result can be extended in the same way that Theorem 2.5 was extended in its corollary.

3. One-parameter families of chance variables with the property $\varepsilon$. In this section, we shall apply the results of $\$ 2$ to the special case in which the chance variables $\left\{x_{t}(\omega)\right\}$ defined in $\$ 2$ have the property $\varepsilon$. $\dagger$ An important example of the kind of process being considered here is the following. Let $E x_{t}$ exist for all $t \geqq 0$, and suppose that $E x_{t} \equiv 0$; suppose also that if $0 \leqq t_{0}<\cdots<t_{n}$, the chance variables

$$
x_{t_{1}}-x_{t_{0}}, \cdots, x_{t_{n}}-x_{t_{n-1}}
$$

are mutually independent. The chance variables $\left\{x_{t}(\omega)\right\}, t \geqq 0$, then have the property $\varepsilon$ if $x_{0}$ is set identically 0 at $t=0$. In this case, the qualitative characteristics of the random function $x_{t}$, as given by P. Lévy [6, 7], are well known: $x_{t}$ can be considered continuous except for discontinuities of the first kind (jumps), or, in the terminology of the present paper, there is a space $\Omega$

$\dagger$ Ville [9, pp. 111-130] has discussed families of non-negative chance variables, depending on the parameter $t$ ranging from 0 to $+\infty$, with the property $\varepsilon$. His discussion of the meaning of a continuous process, and of generalized upper bounds is somewhat obscure. 
of a stochastic process with the given $P^{*}$-measure, whose functions $x(t)$ are continuous except for jumps. $\dagger$ It will be seen below that much of this regularity is true in the more general case now under discussion.

Suppose that the chance variables $\left\{x_{t}(\omega)\right\}$ have the property $\mathcal{E}$. Let $t_{n} \rightarrow t$ and suppose that the sequence $\left\{t_{n}\right\}$ is monotone increasing. Then the chance variables $x_{1}, x_{2}, \cdots, x^{\prime}$, with $x_{n}=x\left(t_{n}\right), x^{\prime}=x(t)$ satisfy the hypotheses of Theorem 1.4 , so $\lim _{n \rightarrow \infty} x\left(t_{n}\right)=x_{l}(t)$ exists, with probability 1 , and the variables $x\left(t_{1}\right), x\left(t_{2}\right), \cdots, x_{l}(t), x(t)$ have the property $\varepsilon$. The limit $x_{l}(t)$ is independent of the sequence $\left\{t_{n}\right\}$ (neglecting $\Omega^{*}$-sets of zero $P^{*}$-measure), since two sequences can be combined into a single one, and evidently $t_{n} \uparrow t$ implies that $x\left(t_{n}\right) \rightarrow x_{l}(t)$ with probability 1 , even if the sequence $\left\{t_{n}\right\}$ is not monotone. Similarly, using Theorem 1.2, $t_{n} \downarrow t$ implies that $\lim _{n \rightarrow \infty} x\left(t_{n}\right)=x_{r}(t)$ exists, with probability 1 , and is independent of the sequence $\left\{t_{n}\right\}$, neglecting $\Omega^{*}$ sets of zero $P^{*}$-measure. By Theorem $2.7, x_{l}(t)=x_{r}(t)=x(t)$ with probability 1 , if $t$ is not in some set $D$ which is at most denumerable. It also follows from Theorem 1.2 that there is a chance variable $x(-\infty)$ such that if $t_{n} \rightarrow-\infty$ then $x\left(t_{n}\right) \rightarrow x(-\infty)$ with probability 1 . The family of chance variables $\{x(t)\}$ $=\left\{x_{t}(\omega)\right\}$ augmented by the newly defined $x_{l}(t), x_{r}(t), x(-\infty)$, and ordered in the natural way, has the property $\varepsilon$. By the corollary to Theorem 0.2, $E|x(t)|$ is a monotone non-decreasing function of $t$. If $E|x(t)|$ is a bounded function, Theorem 1.3 shows that there is a chance variable $x(+\infty)$ such that if $t_{n} \rightarrow+\infty, x\left(t_{n}\right) \rightarrow x(+\infty)$, with probability 1 . Theorem 0.3 states that the chance variables $x_{t}(\omega)$, for $t$ in any set bounded on the right, are uniformly integrable.

Theorem 3.1. Let $\left\{x_{t}\right\},-\infty<t<+\infty$, be chance variables with the property $\mathcal{E}$. Define $\Lambda, \mathrm{M}$ by

$$
\Lambda=\left\{U_{I}\left[x_{t}\right]>k\right\}, \quad \mathbf{M}=\left\{L_{I}\left[x_{t}\right]<k\right\},
$$

where $I$ is the interval $a \leqq t \leqq b$. Then if $s \geqq b$,

$$
\int_{\Lambda} x_{s} d P \geqq k P(\Lambda), \quad \int_{\mathrm{M}} x_{s} d P \leqq k P(\mathrm{M}) \text {. }
$$

If $E\left|x_{b} \log \right| x_{b}||<\infty, E\left\{U_{I}\left[\left|x_{t}\right|\right]\right\}<\infty$.

We have shown that

$$
U_{I}\left[x_{t}\right]=\underset{j \geqq 1}{\text { L.U.B. }} x_{s_{j}}, \quad L_{I}\left[x_{t}\right]=\underset{j \geqq 1}{\text { G.L.B. }} x_{s_{j}}
$$

$\dagger$ Cf. Doob [1, pp. 134-135], and also [2] where the above specific case, involving $E x_{t}$ is discussed. The hypothesis of the existence of $E x_{t}$ is unnecessary to obtain the above regularity properties of $x_{t}$; it is the independence condition that is essential.

$\ddagger$ Cf. Ville [9, pp. 113-119]. 
for a suitably chosen sequence $s_{j}$ in the interval $I$. By Theorem 1.1, if

it follows that

$$
\Lambda_{n}=\left[\underset{j \leqq n}{\operatorname{L} . \mathrm{U} . \mathrm{B} .} x_{s_{j}} \geqq k+1 / n\right]
$$

$$
\int_{\Lambda_{n}} x_{s} d P \geqq k P\left(\Lambda_{n}\right)
$$

$s \geqq b$.

If $n$ becomes infinite, this becomes the first inequality in (3.1.1), and the second is proved in the same way. If $E\left|x_{b} \log \right| x_{b}||<\infty$, there is, according to Theorem 1.5, a constant $K$, depending only on this expectation, such that

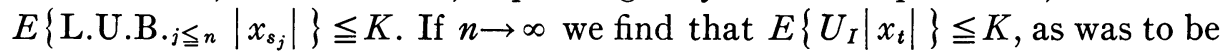
proved.

If the chance variables $\left\{x_{t}(\omega)\right\}$ have the property $\varepsilon$, we have seen that $t_{n} \rightarrow t$ implies that $x\left(t_{n}\right) \rightarrow x(t)$ with probability 1 , unless $t$ is in an exceptional set which is at most denumerable. There is then a measurable process. $\dagger$ According to Theorem 2.5, there is a quasi-separable measurable process. By Theorem 3.1, if $I$ is any finite interval, $U_{I}[|x(t)|]<\infty$, with probability 1 . Then it is unnecessary to use infinite-valued functions in discussing quasi-separable processes. Let $D_{1} \subset D$ be the (at most denumerable) set of $t$-values where $x(t), x_{r}(t)$ are different on $\Omega^{*}$-sets of positive measure. The main result of this section is that there is a space $\Omega$ of a stochastic process with the given probability measure whose elements are continuous except possibly on a denumerable $t$-set, and everywhere continuous on the right, except perhaps at points of $D_{1}$ (the limit $\lim _{t^{\prime} \downarrow t} x\left(t^{\prime}\right)$ existing even on $D_{1}$ ). In order to prove this, we shall need several preliminary results.

LEMMA 3.2. Let $u_{\alpha}(\omega)$ be a measurable function of $\omega$, where $\omega$ varies on some space on which a measure function is defined. The subscript $\alpha$ ranges through the ordinals of the first and second classes. Suppose that for each $\omega$ if $\alpha_{2}>\alpha_{1}$, $u_{\alpha_{2}}(\omega)>u_{\alpha_{1}}(\omega)$ unless $u_{\beta}(\omega)=u_{\alpha_{1}}(\omega)$ for all $\beta>\alpha$. Let $u(\omega)=$ L.U.B. ${ }_{\alpha \geqq 1} u_{\alpha}(\omega)$. Then there is a subscript $\nu$ such that $u_{\nu}(\omega)=u(\omega)$ for almost all $\omega$.

We shall only need this lemma if the $\left\{u_{\alpha}(\omega)\right\}$ are uniformly bounded and if the $\omega$-measure is finite-valued, so only this case will be proved, but the extension to the general case can easily be done. Let $\epsilon$ be any positive number. We shall prove first that there is a subscript $\beta=\beta_{\epsilon}$ such that if $\gamma>\beta, u_{\gamma}-u_{\beta}<\epsilon$ except possibly on a set (depending on $\gamma$ ), of measure less than $\epsilon$. If this is not so there are pairs $\left(\beta_{1}, \gamma_{1}\right),\left(\beta_{2}, \gamma_{2}\right), \cdots$ with $\beta_{1}<\gamma_{1}<\beta_{2}<\gamma_{2}<\cdots$ such that $\mu_{\gamma_{n}}(\omega)-\mu_{\beta_{n}}(\omega) \geqq \epsilon$ on an $\omega$-set $\Lambda_{n}$ of measure not less than $\epsilon$. The set of points $\Lambda$ in infinitely many $\Lambda_{n}$ has measure at least $\epsilon$. Now this is impossible, since

$\dagger$ Doob [1, p. 118]. 
then $u(\omega)=+\infty$ on $\Lambda_{n}$, and we have supposed that the $u_{\alpha}$ are uniformly bounded. There are therefore subscripts $\beta_{1}, \beta_{2}, \cdots, \beta_{1}<\beta_{2}<\cdots$ such that (we are choosing $\epsilon$ successively as $1 / 2,1 / 3, \ldots$ in the above result) if $\gamma>\beta_{n}$

$$
u_{\gamma}-u_{\beta_{n}}<1 / n \text {, }
$$

except possibly on a set of measure less than $1 / n$. The sequence $\left\{\mu_{\beta_{n}}(\omega)\right\}$ is monotone; let $\lim _{n \rightarrow \infty} u_{\beta_{n}}=u_{\infty}$. Let $\nu$ be any ordinal of the second class, beyond $\beta_{1}, \beta_{2}, \cdots$. Then $\mu_{\nu} \geqq \mu_{\beta_{n}}, n \geqq 1$, so $u_{\nu} \geqq u_{\infty}$ for almost all $\omega$. On the other hand, $u_{\nu} \leqq u_{\beta_{n}}+1 / n$, except possibly on a set of measure not greater than $1 / n$. Therefore $u_{\nu} \leqq u_{\infty}$ for almost all $\omega$. It follows that $u_{\nu}=u_{\infty}$ for almost all $\omega$ and, since $\nu$ was any ordinal of the second class beyond $\beta_{1}, \beta_{2}, \cdots, u_{\nu+1}=u_{\infty}$, for almost all $\omega$ also. Thus except for an $\omega$-set $\Lambda_{0}$ of zero measure, $u_{\nu+1}=u_{\infty}$. This implies that if $\omega \notin \Lambda_{0}, u_{\nu}=u_{\nu+1}=\cdots=u$, as was to be proved.

Leммa 3.3. Suppose that $P^{*}$-measure has the property that for almost all $t$, $t_{n} \rightarrow t$ implies that $x\left(t_{n}\right) \rightarrow x(t)$ with probability 1 . There is then a space $\Omega$ of a quasi-separable measurable process. Let $\phi(\omega)$ be an $\Omega$-measurable function and let $\phi_{n}(\omega)=\nu / n$, where

$$
(\nu-1) / n \leqq \phi(\omega)<\nu / n, \quad \nu=0, \pm 1, \cdots, n=1,2, \cdots .
$$

Then $x\left[s+\phi_{n}(\omega)\right], x[s+\phi(\omega)] \dagger$ are measurable $(s, \omega)$-functions $(\omega \varepsilon \Omega)$ and $x\left[s+\phi_{n}(\omega)\right] \rightarrow x[s+\phi(\omega)]$ almost everywhere on $(s, \omega)$-space $(\omega \varepsilon \Omega)$.

According to Theorem 2.4, there is a quasi-separable process with the given $P^{*}$-measure. The hypotheses have been shown to imply that any quasiseparable process is measurable. $\ddagger$ Let $\Omega$ be the space of a quasi-separable measurable process. To show that $x[s+\phi(\omega)]$ is $(s, \omega)$-measurable $(\omega \varepsilon \Omega)$, we must show that for any $k,\{x[s+\phi(\omega)]>k\}$ is an $(s, \omega)$-measurable set. Since $x_{s}(\omega)$ is $(s, \omega)$-measurable $(\omega \varepsilon \Omega)$, because of the measurability of the process with space $\Omega$, we need only show that if $\Lambda$ is an $(s, \omega)$-measurable set, the points $(s, \omega)$ with $(s+\phi(\omega), \omega) \varepsilon \tilde{\Lambda}$ constitute an $(s, \omega)$-measurable set. Denote this image of $\tilde{\Lambda}$ by $\tilde{\Lambda}_{1}$. We shall show that the transformation $\tilde{\Lambda} \rightarrow \tilde{\Lambda}_{1}$ takes measurable sets into measurable sets. Suppose first that $\tilde{\Lambda}=I \times \Lambda$ : the direct product of an $s$-interval $a \leqq s \leqq b$ and a measurable $\Omega$-set $\Lambda$. Then $\tilde{\Lambda}_{1}$ is a simple ordinate set: the $(s, \omega)$ points "over" $\Lambda$ determined by the inequalities

$$
a-\phi(\omega) \leqq s \leqq b-\phi(\omega),
$$

and an ordinate set of this type is known to be measurable. It follows that if $\Lambda$

$\dagger$ The function $x[s+\phi(\omega)]$ is the function $x_{\sigma}(\omega)$, where $\sigma=s+\phi(\omega)$, and $x\left[s+\phi_{n}(\omega)\right]$ is defined similarly.

$\ddagger$ Doob [1, p. 118]. 
is in the Borel field $\mathcal{F}$ of sets determined by the sets which are direct products of $s$-intervals and $\Omega$-measurable sets, the corresponding $\tilde{\Lambda}_{1}$ is $(s, \omega)$-measurable. In particular suppose that $\tilde{\Lambda} \varepsilon \mathcal{F}$ and that $\tilde{\Lambda}$ has measure 0 . Then for almost all $\omega_{0}$ the $s$-set $\left\{\left(s, \omega_{0}\right) \varepsilon \tilde{\Lambda}\right\}$ is of measure 0 . This same fact will certainly be true of the $s$-set $\left\{\left(s+\phi\left(\omega_{0}\right), \omega_{0}\right) \varepsilon \tilde{\Lambda}\right\}$ so the set $\tilde{\Lambda}_{1}$ will have measure 0 . Since any $(s, \omega)$-measurable set of measure 0 can be included in some set of $\mathcal{\exists}$ which is of measure 0 , it follows that whenever $\tilde{\Lambda}$ is measurable and has measure 0 , the same is true of $\tilde{\Lambda}_{1}$. Now the most general $(s, \omega)$-measurable set $\tilde{\Lambda}$ differs from some set $\tilde{M}$ of $\mathcal{F}$ at most by a set of measure 0 , so the corresponding $\tilde{\Lambda}_{1}$ will differ from $\tilde{M}_{1}$ by at most a set of measure $0: \tilde{\Lambda}_{1}$ is measurable, as was to be proved. We have thus proved that $x[s+\phi(\omega)]$ (and therefore $\left.x\left[s+\phi_{n}(\omega)\right]\right)$ is $(s, \omega)$-measurable $(\omega \varepsilon \Omega)$. According to Theorem 2.7, if $x(t) \varepsilon \Omega$, $x(t)$ is continuous for almost all $t$, with probability 1 . Then if $\omega$ is fixed, $x\left[s+\phi_{n}(\omega)\right] \rightarrow x[s+\phi(\omega)]$ for almost all $s$, with probability 1 . This implies that $x\left[s+\phi_{n}(\omega)\right] \rightarrow x[s+\phi(\omega)]$ for almost all $(s, \omega)$, which is the final conclusion of the lemma.

LEMmA 3.4. Let $u(\omega)$ be an $\omega$-measurable function, defined on the space $\Omega$ of a stochastic process whose chance variables $\left\{x_{t}(\omega)\right\}$ have the property $\varepsilon$. Suppose that $u(\omega)$ takes on only a finite number of values: $u(\omega)=\lambda_{j}$ on $\Lambda_{j}$, $j=1,2, \cdots$. Suppose that for each $j,\left\{u(\omega)=\lambda_{j}\right\}$ is an $\omega$-set depending on $\omega$ : $x(t)$ for $t<\lambda_{j}$. Then the chance variables $\left\{y_{t}\right\}, y_{t}=x[t+u(\omega)]$ (that is, $y_{t}=x_{\sigma}(\omega)$, $\sigma=t+u(\omega))$, have the property $\mathcal{E}$, for $t>0$.

To prove this fact, we need only show that if $\mathbf{M}$ is a $P^{*}$-measurable set depending on the $y(t)$ for $t=t_{1}, \cdots, t_{\nu}, 0<t_{1}<\cdots<t_{\nu}<t_{\nu}+h$, then

$$
\int_{\mathbf{M}} y_{t_{\nu}+h} d P=\int_{\mathbf{M}} y_{t_{\nu}} d P
$$

Now

$$
\int_{\mathbf{M}} y_{t_{\nu}+h} d P=\sum_{j} \int_{\Lambda_{j} \cdot \mathbf{M}} y_{t_{\nu}+h} d P=\sum_{j} \int_{\Lambda_{j} \cdot \mathbf{M}} x\left(t_{\nu}+h+\lambda_{j}\right) d P .
$$

The conditions on the $y\left(t_{i}\right)$ determining $\mathbf{M}$ are, on $\Lambda_{j}$, conditions on $x\left(t_{i}+\lambda_{j}\right)$, $i=1, \cdots, \nu$, so $\Lambda_{j} \cdot \mathbf{M}$ is a set depending on $t$-values for $t \leqq t_{\nu}+\lambda_{j}$. Because of the fact that

$$
E\left[x_{t}, t \leqq t_{\nu}+\lambda_{j} ; x_{t_{\nu}+\lambda_{j}+h}\right]=x_{t_{\nu}+\lambda_{j}},
$$

with probability 1 (Theorem 0.1 ), we find that

$$
\int_{\Lambda_{j} \cdot M} x\left(t_{\nu}+\lambda_{j}+h\right) d P=\int_{\Lambda_{j} \cdot M} x\left(t_{\nu}+\lambda_{j}\right) d P=\int_{\Lambda_{j} \cdot M} y_{t \nu} d P .
$$


Summing over $j$ gives

$$
\int_{\mathbf{M}} y_{t_{\nu}+h} d P=\int_{\mathbf{M}} x\left(t_{\nu}+u+h\right) d P=\int_{\mathbf{M}} y_{t_{\nu}} d P,
$$

or (3.4.1), as was to be proved.

LEмmA 3.5. Let $u(\omega)$ be a bounded $\omega$-measurable function, defined on the space $\Omega$ of a stochastic process whose chance variables $x_{t}(\omega)$ have the property $\varepsilon$. Suppose that for each number $c,\{u(\omega) \geqq c\}$ is an $\omega$-set depending on $\omega: x(t)$ for $t<c$. Let $S$ be any everywhere dense denumerable set. Then $\lim _{8+0} x[s+u(\omega)] \dagger$ $(s$ \& $S)$ exists with probability 1 .

Eet $u_{n}(\omega)=\nu / n$ where

$$
(\nu-1) / n \leqq u(\omega)<\nu / n, \quad \nu=0, \pm 1, \cdots, n=1,2, \cdots .
$$

Then $u_{n}(\omega)$ satisfies the hypotheses of Lemma 3.4. Let $y_{n}(s)=x\left[s+u_{n}(\omega)\right]$, $y(s)=x[s+u(\omega)]$. We show first that, for fixed $n$ and $s>0$, if $T>s+$ L.U.B. ${ }_{\omega} u(\omega)$, then

$$
E\left[y_{n}(s) ; x(T)\right]=y_{n}(s),
$$

with probability 1 , that is, that the ordered pair of chance variables $y_{n}(s)$, $x(T)$ has the property $\varepsilon$. Let $\Lambda$ be any $\omega$-set depending on $y_{n}$, that is, determined by some condition imposed on $y_{n}$. It must be shown that

$$
\int_{\Lambda} y_{n}(s) d P=\int_{\Lambda} x(T) d P .
$$

Let $\Lambda_{n, j}=\left\{u_{n}=j / n\right\}$. Then

$$
\int_{\Lambda} y_{n}(s) d P=\sum_{j} \int_{\Lambda \cdot \Lambda_{n, j}} x(s+j / n) d P .
$$

Now the $\omega$-set $\Lambda_{n, j}$ depends only on $t$-values less than $j / n$, and on $\Lambda_{n, j}$ the condition on $y_{n}$ determining $\Lambda$ becomes one on $x(s+j / n)$, so $\Lambda \cdot \Lambda_{n, j}$ depends only on $t$-values not greater than $s+j / n$. This means that, using the property $\varepsilon$,

$$
\int_{\Lambda} y_{n}(s) d P=\sum_{j} \int_{\Delta \cdot \mathbf{\Delta}_{n, j}} x(T) d P=\int_{\Lambda} x(T) d P,
$$

or (3.5.1), as was to be shown. Now if $n$ varies, it follows that the $y_{n}(s)$ are uniformly integrable, from the proof of Theorem 0.3 : in fact, the proof of that theorem did not use the full property $\varepsilon$, but only the fact that, for

$\dagger$ The function $x[s+u(\omega)]$ is the function $x_{\sigma}(\omega)$, where $\sigma=s+u(\omega)$. 
$t \leqq t_{0}, E\left[x_{t} ; x_{t_{0}}\right]=x_{t}$ with probability 1 . This corresponds to the fact that $E\left[y_{n} ; x(T)\right]=y_{n}, n=1,2, \cdots$, with probability 1 .

Having these preliminary facts in mind, we proceed to prove Lemma 3.1. We enlarge $S$ to include the rational numbers, and so that $S$ contains the number $s_{1}+s_{2}$ if it contains $s_{1}$ and $s_{2}$. Let $\delta_{1}, \delta_{2}$ be positive numbers, with $\delta_{1}<\delta_{2}$. According to Lemma 3.4, the $y_{n}(s)$ for $s>0$ ( $n$ fixed) have the property $\varepsilon$. Then it can be verified at once that the chance variables $y_{n}(s)-y_{n}\left(\delta_{1}\right)$, for $s>\delta_{1}$ ( $n$ fixed) have the property $\varepsilon$. Using Theorem 3.1, we find that

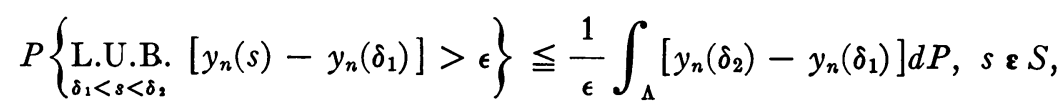

where $\Lambda$ is the set on the left, and

$$
P\left\{\underset{\delta_{1}<s<\delta_{2}}{\text { G.L.B. }}\left[y_{n}(s)-y_{n}\left(\delta_{1}\right)\right]<-\epsilon\right\} \leqq \frac{1}{\epsilon} \int_{M}\left[y_{n}\left(\delta_{2}\right)-y_{n}\left(\delta_{1}\right)\right] d P, s \varepsilon S,
$$

where $M$ is the set on the left. Then surely

$$
\begin{gathered}
P\left\{\begin{array}{c}
\text { L.U.B. } \left.\left[y_{n}(s)-y_{n}\left(\delta_{1}\right)\right]>\epsilon\right\} \\
\delta_{1}<s<\delta_{2}
\end{array}\right. \\
P\left\{\frac{1}{\epsilon} \int\left|y_{n}\left(\delta_{2}\right)-y_{n}\left(\delta_{1}\right)\right| d P,\right. \\
P\left\{\begin{array}{c}
\text { G.L.B. } S, \\
\delta_{1}<s<\delta_{2}
\end{array}\right.
\end{gathered}
$$

so that

$$
P\left\{\underset{\delta_{1}<s<\delta_{2}}{\text { L.U.B. }}\left|y_{n}(s)-y_{n}\left(\delta_{1}\right)\right|>\epsilon\right\} \leqq \frac{2}{\epsilon} \int\left|y_{n}\left(\delta_{2}\right)-y_{n}\left(\delta_{1}\right)\right| d P, s \varepsilon S,
$$

which implies that

$$
\begin{aligned}
& P\left\{\underset{\delta_{1}<s^{\prime}, s^{\prime \prime}<\delta_{2}}{\text { L.U.B. }}\left|y_{n}\left(s^{\prime}\right)-y_{n}\left(s^{\prime \prime}\right)\right|>2 \epsilon\right\} \leqq \frac{2}{\epsilon} \int\left|y_{n}\left(\delta_{2}\right)-y_{n}\left(\delta_{1}\right)\right| d P, \\
& s^{\prime}, s^{\prime \prime} \varepsilon S \text {. }
\end{aligned}
$$

Now if $\xi_{n}$ denotes the quantity on the left in (3.5.9),

$$
\begin{aligned}
\xi_{n} & =\sum_{\nu} P\left\{\underset{\delta_{1}<s^{\prime}, s^{\prime \prime}<\delta_{2}}{\text { L.U.B. } \mid}\left|x\left(s^{\prime}+\nu / n\right)-x\left(s^{\prime \prime}+\nu / n\right)\right|>2 \epsilon ; u_{n}=\nu / n\right\} \\
(3.5 .10) & =\sum_{\nu} P\left\{\underset{\delta_{1}+\nu / n<s^{\prime}, s^{\prime \prime}<\delta_{2}+\nu / n}{\text { L.U.B. }}\left|x\left(s^{\prime}\right)-x\left(s^{\prime \prime}\right)\right|>2 \epsilon ; u_{n}=\nu / n\right\}, \\
& \geqq P\left\{\underset{\delta_{1}+u+1 / n<s^{\prime}, s^{\prime \prime}<\delta_{2}+u}{\text { L.U.B. }}\left|x\left(s^{\prime}\right)-x\left(s^{\prime \prime}\right)\right|>2 \epsilon\right\},
\end{aligned}
$$


It is easily verified that all the sets involved are $\omega$-measurable. When $n$ becomes infinite, we obtain

(3.5.11) $\liminf _{n \rightarrow \infty} \xi_{n} \geqq P\left\{\underset{\delta_{1}+u<s^{\prime}, s^{\prime \prime}<\delta_{2}+u}{\text { L.U.B. }}\left|x\left(s^{\prime}\right)-x\left(s^{\prime \prime}\right)\right|>2 \epsilon\right\}, \quad s^{\prime}, s^{\prime \prime} \varepsilon S$.

It was shown in Lemma 3.3 that $y_{n}(s) \rightarrow y(s)$ for almost all $(s, \omega)$. Then except for some $s$-set $e$ of measure $0, y_{n}(s) \rightarrow y(s)$ for almost all $\omega$. Choose $\delta_{1}, \delta_{2}$ not in $e$. Combining (3.5.9), (3.5.10), (3.5.11), we find that

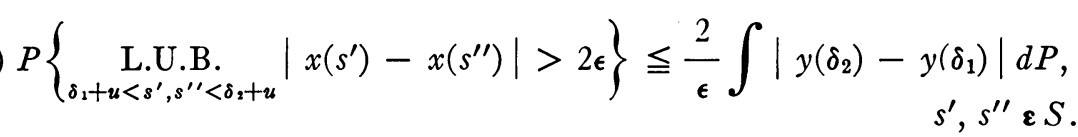

We can integrate to the limit because the $y_{n}$ have been shown to be uniformly integrable. Now let $\delta_{1} \rightarrow 0$ along a sequence of values remaining out of the set $e$. By Theorem 1.2, $y(\delta)$ approaches a limit with probability 1 , say $y(0+)$, and we can integrate term by term, so that

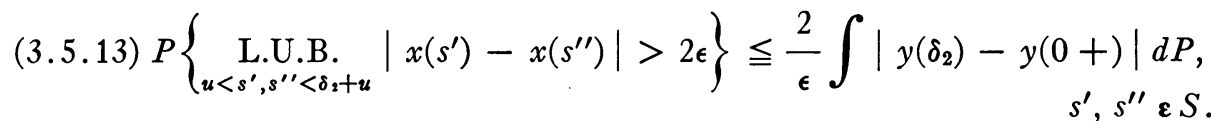

If now $\delta_{2} \rightarrow 0$ along the same sequence of values, the integral goes to 0 . This means that the oscillation of $x(t)$ at $t=u(\omega)$ on the right (only considering the function $x(t)$ defined on $S$ ) is not greater than $2 \epsilon$, with probability 1 . Since $\epsilon$ is arbitrary, the lemma follows at once from this fact.

THEOREM 3.6. Suppose that the chance variables $\left\{x_{t}\right\}$ have the property $\mathcal{E}$. Let $D_{1}$ be the (at most denumerable) set of $t$-values for which $x(t), x_{r}(t)$ are not identical with probability 1 . There is a space $\Omega$ of a quasi-separable measurable stochastic process whose elements $x(t)$ have the following properties: each element $x(t)$ is continuous except possibly at a denumerable set of $t$-values (which may vary with the element $x(t)) ; \lim _{h \downarrow 0} x(t+h)$ exists for all $t$, and the limit is $x(t)$ if $t \notin D_{1} ; \lim _{h \downarrow 0} x(t+h)=x_{r}(t), \lim _{h} \uparrow_{0} x(t+h)=x_{l}(t)$ with probability 1 , at each t-value. $\dagger$

The results obtained in this theorem are less strong than those obtained in the case of a differential process, discussed above, in which the increments of $x(t)$ in nonoverlapping intervals are independent chance variables. In the differential case, the only discontinuities are jumps, whereas here the elements $x(t)$ can apparently have complicated discontinuities on the left (of probability 0 at any fixed $t$-value, however, except where $x_{l}(t), x(t)$ are not

$\dagger$ The exceptional $\Omega$-set depends on $t$. It will be remembered that except for a denumerable $t$-set, $x_{l}(t)=x_{r}(t)=x(t)$ with probability $1(t$ fixed $)$. 
equal with probability 1 , and there the discontinuity is a jump, with probability 1). Some such difference might have been suspected, since the property $\varepsilon$ is essentially unsymmetric in $t$.

Proof of Theorem 3.6. Let $S$ be any everywhere dense denumerable set, and let $\epsilon$ be any positive number. Consider the $t$-set $E[x(t)]$ where the oscillation of $x(t)(t \varepsilon S)$ is not less than $\epsilon$. This is a closed set. The $\omega$-set

$$
\prod_{\nu=1}^{\infty} \sum_{\left|s^{\prime}-s^{\prime \prime}\right|<1 / \nu, a-1 / \nu<8^{\prime}, s^{\prime \prime}<b+1 / \nu}\left\{\left|x\left(s^{\prime}\right)-x\left(s^{\prime \prime}\right)\right| \geqq \epsilon-1 / \nu\right\}, s^{\prime}, s^{\prime \prime} \varepsilon S,
$$

is the set of elements $x(t)$ for which $E$ has at least one point in $a \leqq t \leqq b$. This set is evidently $P^{*}$-measurable. Let $k$ be any positive integer. For each $x(t)$ define $u[x(t)]$ as the first value of $t$ with $-k \leqq t \leqq k$ in $E[x(t)]$ at which the discontinuity on the right is greater than $0 ; \dagger$ if there is no such point, let $u=k$. The points $s$ of $E[x(t)]$ with $-k \leqq s<u[x(t)]$ define a set $F[x(t)]$ with the following properties: there is no sequence $s_{1}, s_{2}, \cdots$ in $F[x(t)]$ with $s_{1}>s_{2}>\cdots ; F[x(t)]$ is at most denumerable. Then if $F[x(t)]$ is not empty, and is ordered from left to right, it is a well-ordered set, whose ordinal number is in the first or second class. If $\alpha$ is any ordinal number in the first or second class, define $u_{\alpha}[x(t)]$ as the $\alpha$ th point of $F[x(t)]$ (or $u_{\alpha}=u$, if there is no $\alpha$ th point). Then if $\beta>\alpha, u_{\beta}[x(t)]>u_{\alpha}[x(t)]$ unless $u_{\alpha}[x(t)]=u_{\alpha+1}[x(t)]$ $=\cdots=u[x(t)]$, and for each function $x(t)$ there will be an $\alpha$ such that the above equalities are true. Concluding this preliminary discussion, we come to the proof of the theorem, which we shall divide into three parts.

I. Let $k_{1}, k_{2}$ be any numbers with $k_{1}<k_{2}$. Let $v[x(t)]$ be the first point of $E[x(t)]$ in the interval $k_{1} \leqq t \leqq k_{2}$, or if there is no such point let $v[x(t)]=k_{2}$. Then we prove that

(i) $v[x(t)]$ is a measurable function of $\omega$,

(ii) the $\omega$-set $v \geqq c$, for any number $c$, depends only on $t$ for $t<c$, and

(iii) the oscillation of $x(t)(t \varepsilon S)$ on the right at $v[x(t)]$ is 0 .

To prove (i) it is sufficient to note that $k_{1} \leqq v \leqq k_{2}$ and that the (measurable) $\omega$-set (3.6.1) with $a=k_{1}, k_{1}<b<k_{2}$, is the set $\{v \leqq b\}$. To prove (ii) we note first (from (3.6.1) with $a=k_{1}, b=c-\eta$ ) that the $\omega$-set $\{v \leqq c-\eta\}$ $\left(k_{1}<c<k_{2}\right)$ depends only on $t$-values less than $c-\eta / 2$, for any $\eta>0$. Letting $\eta \rightarrow 0$, we find that the $\omega$-set $\{v<c\}$ and therefore also $\{v \geqq c\}$ depends only on $t$-values less than $c$. It then follows that (ii) holds for all $c$. Lemma 3.5 can now be applied to show that $\lim _{s \downarrow v} x(s)(s \varepsilon S)$ exists with probability 1 , that is, that (iii) is true.

$\dagger$ The discontinuity on the right at $t_{0}$ is defined as $\lim _{\delta \rightarrow 0}$ L.U.B. $t_{0}<s^{\prime}, s^{\prime \prime}<t_{0}+\delta\left|x\left(s^{\prime}\right)-x\left(s^{\prime \prime}\right)\right|$ $\left(s^{\prime}, s^{\prime \prime} \varepsilon S\right)$. 
II. We next prove that for each $\alpha$

(i) $u_{\alpha}[x(t)]$ is a measurable function of $\omega$ and

(ii) $\left\{u_{\alpha}[x(t)] \geqq c\right\}$, for any number $c$, depends only on $t$-values for $t<c$.

The proof will be given by transfinite induction. Consider the case $\alpha=1$. Let $v[x(t)]$ be the first point of $E[x(t)]$ in the interval $-k \leqq t \leqq k$, or let $v=k$ if there is no such point. Then I shows that $v=u_{1}$ with probability 1 , and that $u_{1}$ has the properties (i) and (ii) since $v$ has. Suppose that (i) and (ii) are true for some $\alpha$. We shall show that they are then true for $\alpha+1$. Let $w[x(t)]$ be the first point of $E[x(t)]$ in the interval $u_{\alpha}[x(t)]<t \leqq k$; let $w=k$, if there is no such point or if $u_{\alpha}=k$. We can obtain $w$ as follows: for each rational number $r<k$ determine the $\omega$-set $\left\{u_{\alpha}<r\right\}$; on this set $w$ is the first point of $E[x(t)]$ beyond $r$, and not greater than $k$, or $w=k$ if there is no such point; if $u_{\alpha}=k, w=k$ also. This makes it obvious, using I, that $w=u_{\alpha+1}$ with probability 1. Properties (i), (ii) are true for $u_{\alpha+1}$ since they are true for $w$ (as can be seen using I and the fact that (i), (ii) are known to be true for $u_{\alpha}$ ). To finish the induction proof of (i), (ii), we must show that if $\alpha_{1}<\alpha_{2}<\cdots$, and if $\alpha$ is the first ordinal beyond the $\alpha_{j}$, then (i), (ii) are true for $u_{\alpha}$ if they are supposed true for $u_{\alpha_{1}}, u_{\alpha_{2}}, \cdots$. Let $w=\lim _{j \rightarrow \infty} u_{\alpha_{j}}$. Then $w$ is a $P^{*}$-measurable function, $w \leqq u$, and if $w[x(t)]<k, w[x(t)]$ is certainly a point of $E[x(t)]$. Moreover, since (ii) is true for $u_{\alpha_{1}}, u_{\alpha_{2}}, \cdots$, it follows easily that (ii) is true for $w[x(t)]$. Lemma 3.5 now shows that $x(t)$ has 0 oscillation on the right $(t \varepsilon S)$ at $w$, with probability 1 , so that $w[x(t)]=u_{\alpha}[x(t)]$, with probability 1 . Therefore $u_{\alpha}$ has the properties (i) and (ii), since $w$ has. We have thus proved that (i), (ii) hold for all $\alpha$.

III. The functions $\left\{u_{\alpha}(\omega)\right\}$ satisfy the hypotheses of Lemma 3.2, so there is an index $\nu$ such that $u_{\nu}=$ L.U.B. ${ }_{\alpha} u_{\alpha}=u$ except possibly on an $\omega$-set of measure 0 . It is impossible that $u_{\nu}=u<k$ on an $\omega$-set of positive measure, because $u_{\nu}$ is a point with 0 oscillation of $x(t)(t \varepsilon S)$ on the right, with probability 1 (Lemma 3.5), whereas $u$ is by hypothesis a point where the oscillation on the right is positive, if $u<k$. Then $u_{\nu}=u=k$ almost everywhere on $\Omega^{*}$. The indices $\alpha \leqq \nu$ are denumerable, at most, so we have proved that if an $\omega$-set of measure 0 is excluded, $\lim _{t^{\prime} \downarrow t} x\left(t^{\prime}\right)\left(t^{\prime} \varepsilon S\right)$ exists for all $t$, with $|t| \leqq k$, where the oscillation of $x(t)(t \varepsilon S)$ is at least $\epsilon$. If we apply this result letting $\epsilon$ run through the values $1,1 / 2,1 / 3, \cdots$ and $k$ through the values $1,2, \cdots$, we find that neglecting some $\omega$-set of measure $0, \lim _{t^{\prime} \downarrow_{t}} x\left(t^{\prime}\right)\left(t^{\prime} \varepsilon S\right)$ exists for all $t$. Theorem 2.8 can now be applied to give the conclusion of the present theorem, except that part involving $\lim _{h \downarrow_{0}} x(t+h), \lim _{h \uparrow_{0}} x(t+h)$ ( $t$ fixed), $\lim _{t \rightarrow \infty} x(t), \lim _{t \rightarrow-\infty} x(t)$. We have seen above, in our preliminary discussion of continuous stochastic processes whose chance variables $\left\{x_{t}(\omega)\right\}$ have the property $\varepsilon$, that if $t_{n} \downarrow t, x\left(t_{n}\right) \rightarrow x_{r}(t)$ with probability 1 . This implies that if $S$ 
is any denumerable set, $\lim _{t^{\prime} \downarrow t} x\left(t^{\prime}\right)=x_{r}(t)\left(t^{\prime} \varepsilon S\right)$, with probability $1 \dagger$; so if the process is quasi-separable, $\lim _{h \downarrow 0} x(t+h)=x_{r}(t)$, with probability 1 . The other cases are treated similarly.

THEOREM 3.7. Let $P^{*}$-measure be arbitrary, and let $x$ be any chance variable depending on the $x_{t}$ (that is, a $P^{*}$-measurable function) whose expectation exists. Let $\Omega$ be the space of a stochastic process with this $P^{*}$-measure. Then if $y(t)$ $=E\left[x_{s}, s \leqq t ; x\right], \ddagger$ these conditional expectations can be defined so that for $\omega$ fixed, in $\Omega$, not in some set of probability $0, y(t)$ is continuous except possibly on a denumerable set, and even on this set $\lim _{h \downarrow 0} y(t+h)$ exists; moreover $\lim _{t \rightarrow-\infty} y(t)$ exists, and $\lim _{t \rightarrow+\infty} y(t)=x$.

The chance variables $y(t)$ have the property $\varepsilon$. This theorem follows readily from Theorem 3.6, using the discussion of families of $P^{*}$-measurable functions at the end of $\$ 2$. The fact that the limit as $t \rightarrow+\infty$ is $x$ follows from Theorem 1.4.

\section{BIBLIOGRAPHY}

1. J. L. Doob, Stochastic processes depending on a continuous parameter, these Transactions, vol. 42 (1937), pp. 107-140.

2. - The law of large numbers for continuous stochastic processes, to appear in the Duke Mathematical Journal.

3. J. L. Doob and Warren Ambrose, to appear in the Annals of Mathematics.

4. A. Khintchine, Asymptotische Gesetze der Wahrscheinlichkeitsrechnung, Ergebnisse der Mathematik, vol. 2 , no. 4 .

5. A. Kolmogoroff, Grundbegriffe der Wahrscheinlichkeitsrechnung, Ergebnisse der Mathematik, vol. 2 , no. 3 .

6. P. Lévy, Sur les intégrales dont les éléments sont des variables aléatoires indépendantes, Annali delle Università Toscane, Pisa, (2), vol. 3 (1934), pp. 337-366.

7. —, Sur un précédent mémoire de l'auteur, ibid., (2), vol. 4 (1935), pp. 217-218.

8. — Théorie de l'Addition des Variables Aléatoires, Paris, 1937.

9. Jean Ville, Étude Critique de la Notion de Collectif, Paris, 1939.

10. N. Wiener, The ergodic theorem, Duke Mathematical Journal, vol. 5 (1935), pp. 1-18.

$\dagger$ Doob [1, p. 111].

$\ddagger$ Each conditional expectation is only uniquely defined up to an $\Omega^{*}$-set of measure 0 .

UNIVERSITY OF ILLINOIS,

URBANA, ILL. 